\title{
LXXX. Reflections on the resolution of algebraic equations of the fifth degree
}

\section{G.B. Jerrard}

To cite this article: G.B. Jerrard (1845) LXXX. Reflections on the resolution of algebraic equations of the fifth degree, Philosophical Magazine Series 3, 26:176, 545-574, DOI: $10.1080 / 14786444508645195$

To link to this article: http://dx.doi.org/10.1080/14786444508645195

册 Published online: 30 Apr 2009.

Submit your article to this journal $\sqsubset \pi$

Џ Article views: 2

Q View related articles $\square$ 


\title{
PHILOSOPHICAL MAGAZINE
}

AND

\section{JOURNAI OF SCIENCE.}

\author{
SUPPLEMENT тo VOL. XXVI. THIRD SERIES.
}

LXXX. Reflections on the Resolution of Algebraic Equations of the Fifth Degree. By G. B. Jerrard, Examiner in Mathematics and Natural Philosophy at the University of London*.

SOME years ago, while reflecting on the possibility of disN covering an expression which, consisting of a finite combination of radicals and rational functions, would satisfy the general equation of the fifth degree,

$$
x^{5}+A_{1} x^{4}+A_{2} x^{3}+A_{3} x^{2}+A_{4} x+A_{5}=0,
$$

I was led to a result which seems to indicate the possibility of assigning, in a subsidiary equation of the third degree with respect to $x$,

$$
x^{3}+p_{1} x^{2}+p_{2} x+p_{3}=y
$$

such finite expressions to $p_{1}, p_{2}, p_{3}$, that the equation for $y$ may take the known form

$$
y^{5}+\mathrm{A}_{2}^{\prime} y^{3}+\frac{1}{5} \mathrm{~A}_{2}^{2} y+\mathrm{A}_{5}^{\prime}=0
$$

If $p_{1}, p_{2}, p_{3}$ admit of having the requisite expressions assigned to them, it must, in opposition to what has been advanced by $A$ bel $\dagger$, be possible to discover five finite algebraic expressions capable of satisfying the equation

$$
x^{5}+A_{1} x^{4}+A_{2} x^{3}+A_{3} x^{2}+A_{4} x+A_{5}=0 .
$$

In effect, we might obtain, as we shall see in another place,

or rather the system of equations

$$
x=q_{4}+q_{3} y+q_{2} y^{2}+q_{1} y^{3}+q_{0} y^{4},
$$

$$
\begin{gathered}
x_{1}=q_{4}+q_{3} y_{1}+\cdots+q_{0} y_{1}^{4}, \\
x_{2}=q_{4}+q_{3} y_{2}+\cdots+q_{0} y_{2}^{4} \\
\dot{\bullet} \\
\dot{x_{5}}=q_{4}+q_{3} y_{5}+\dot{\cdot}+q_{0} y_{5}^{4}
\end{gathered}
$$

- Communicated by the Author.

+ A complete exposition of the argument of Abel is given by Sir William R. Hamilton in vol, xviii. part 2 of the Transactions of the Royal Irish Academy.

Phil. Mag. S. 3. No. 176. Suppl. Vol. 26. 
where $x_{1}, x_{2}, \ldots x_{5}$ are the roots of $x^{5}+A_{1} x^{4}+\ldots=0 ; y_{1}$, $y_{2}, \ldots y_{5}$ of $y^{5}+A_{2} y^{3}+\ldots=0$; and where $q_{4}, q_{3}, \ldots q_{0}, y_{1}$, $y_{2}, \ldots y_{5}$ are all of them expressible as known functions of $p_{1}$, $p_{2}, p_{3}$.

Can then $p_{1}, p_{2}, p_{3}$ be found? To bring this question to a decision, we must enter upon an inquiry of great length and intricacy. But it is in the nature of such discussions to unfold in their progress theories which bear upon remote and seemingly unconnected departments of knowledge: and of this truth we shall find in what follows some remarkable exemplifications.

\section{SECTION I.}

1. Now $p_{1}, p_{2}, p_{3}$ must be such that $x^{3}+p_{1} x^{2}+p_{2} x+p_{3}$ may become a root of an equation of the form

$$
y^{5}+\mathrm{A}_{2}^{\prime} y^{3}+\frac{1}{5} \mathrm{~A}_{2}^{\prime} y+\mathrm{A}_{5}^{\prime}=0 ;
$$

or that the expressions

$$
\begin{aligned}
& x_{1}{ }^{3}+p_{1} x_{1}{ }^{2}+p_{2} x_{1}+p_{3}, \\
& x_{2}{ }^{3}+p_{1} x_{2}{ }^{2}+p_{2} x_{2}+p_{3} \\
& \dot{\cdot} \dot{\cdot} \\
& \dot{x_{5}{ }^{3}+p_{1}} \dot{x}_{5}{ }^{2}+\dot{p_{2}} x_{5}+\dot{p_{3}}
\end{aligned}
$$

may become the five roots of that equation. For $x_{1}, x_{2}, \ldots x_{5}$ enter symmetrically into the calculus, and there is consequently nothing to connect one of them rather than another with the $x$ of the expression $x^{3}+p_{1} x^{2}+p_{2} x+p_{3}$.

If then we consider that the roots of the equation for $y$ must, as De Moivre has shown, be generally expressible by

$$
t+u, \quad t t+i^{4} u, \quad i^{2} t+i^{3} u, \quad i^{3} t+\imath^{2} u, \quad i^{4} t+i u,
$$

i, $t^{2}, t^{3}, t^{4}$ denoting the inaginary roots of the binominl equntion $p^{5}-1=0$, we shall be conducted to a system of equations

$$
\left.\begin{array}{l}
x_{\alpha}^{3}+p_{1} x_{\alpha}^{2}+p_{2} x_{\alpha}+p_{3}=t+u, \\
x_{\beta}^{3}+p_{1} x_{\beta}^{2}+p_{2} x_{\beta}+p_{3}=\imath t+\iota^{4} u, \\
x_{\gamma}^{3}+p_{1} x_{\gamma}^{2}+p_{2} x_{\gamma}+p_{3}=\iota^{2} t+\iota^{3} u, \\
x_{\delta}^{3}+p_{1} x_{\delta}^{2}+p_{2} x_{\delta}+p_{3}=\iota^{3} t+\iota^{2} u, \\
x_{s}^{3}+p_{1} x_{s}^{2}+p_{2} x_{t}+p_{3}=\iota^{4} t+\iota u ;
\end{array}\right\} \cdot \cdot
$$

in which $a, \beta, \gamma, \delta, \approx$ represent, in an undetermined or arbitrary order of succession, the five indices $1,2,3,4,5$.

2 . From this system there will arise, as we know from the theory of permutations, 1.2.3.4.5 systems; if instead of 
$\alpha, \beta, \gamma, \delta$, $\varepsilon$, we substitute $1,2,3,4,5$, in all the different arrangements which they can assume. But these 120 systems will be found to furnish only twelve different sets of values for $p_{1}, p_{2}, p_{3^{*}}$ Our first object will be to express $p_{1}, p_{2}, p_{3}$ as functions of $x_{1}, x_{2}, \ldots x_{5}$ without $t$ and $u$.

3. By combining any three of the five equations of the system (a.), we see that we may eliminate $t$ and $u$; and that therefore, if we replace $x_{a}^{3}+p_{1} x_{\alpha}{ }^{2}+\ldots, x_{\beta}{ }^{3}+p_{1} x_{\beta}{ }^{2}+\ldots$, $\ldots x_{\varepsilon}^{3}+p_{1} x_{\varepsilon}^{2}+\ldots$, by $y_{\alpha}, y_{\beta}, \ldots y_{\varepsilon}$, we may arrive at a final equation of the first degree with respect to $y_{\omega}, y_{\beta}, \ldots y_{s}$,

$$
\mu_{\alpha} y_{\alpha}+\mu_{\beta} y_{\beta}+\mu_{\gamma} y_{\gamma}+\mu_{\delta} y_{\delta}+\mu_{\varepsilon} y_{\varepsilon}=0:
$$

where $\mu_{\alpha}, \mu_{\beta}, \ldots \mu_{\varepsilon}$ are functions of, which have no common factor different from 1 , and are such that two of them must arlmit of being equated to zero.

4. It is clear that $\mu_{\alpha}, \mu_{\beta}, \ldots \mu_{\varepsilon}$ will be susceptible of $\frac{5.4 .3}{1.2 .3}$ or ten differently derived sets of values. The ten equations which may thus arise I shall for the moment represent by

$$
\begin{aligned}
& { }^{3} \mu_{\alpha} y_{\alpha}+{ }^{1} \mu_{\beta} y_{\beta}+\ldots+{ }^{1} \mu_{\varepsilon} y_{\varepsilon}=0, \\
& { }^{2} \mu_{\alpha} y_{\alpha}+{ }^{2} \mu_{\beta} y_{\beta}+\ldots+{ }^{2} \mu_{t} y_{\varepsilon}=0 \text {, } \\
& \dot{{ }^{10} \dot{\mu}_{\mu_{\alpha}} y_{\alpha}+{ }^{10} \hat{0}_{\mu_{\beta}} y_{\beta}+. .} \dot{\cdot}+{ }^{10_{\mu_{\varepsilon}}} \dot{y_{\varepsilon}}=0 .
\end{aligned}
$$

All these will belong to the same system.

5. Further, if we consider that $y_{\alpha}+y_{\beta}+y_{\gamma}+y_{\delta}+y_{s}$, which must be equated to zero, will give

$$
\begin{gathered}
x_{\alpha}{ }^{3}+x_{\beta}^{3}+\ldots+x_{z}^{3}+\left(x_{\alpha}^{2}+x_{\beta}^{2}+\ldots+x_{\varepsilon}^{2}\right) p_{1} \\
+\left(x_{a}+x_{\beta}+\ldots+x_{\varepsilon}\right) p_{2}+5 p_{3}=0 ;
\end{gathered}
$$

and that consequently, if denoting

we eliminate $p_{3}$ from the equation

$$
x_{\alpha}^{n}+x_{\beta}^{n}+\ldots+x_{s}^{n} \text { by } \mathrm{S}_{n}
$$

there will result

$$
x_{\tau}^{3}+p_{1} x_{\tau}{ }^{2}+p_{2} x_{\tau}+p_{3}=y_{\tau}
$$

$$
y_{\tau}=x_{\tau}^{3}-\frac{1}{5} S_{3}+\left(x_{\tau}^{2}-\frac{1}{5} S_{2}\right) p_{1}+\left(x_{\tau}-\frac{1}{5} S_{1}\right) p_{2}:
$$

we shall perceive that any two of those ten equations will, if incapable of being made coincident by any transformation and reduction, be sufficient for enabling us to express $p_{1}$ and $p_{2}$ as rational functions of the roots of the original equation.

6 . It is not difficult actually to obtain such a pair of equa$2 \mathrm{O} 2$ 
tions, and to show that those values of $p_{1}, p_{2}$, which satisfy them, will necessarily satisfy every other pair belonging to the same system.

In effect, if introducing an indeterminate multiplier $\lambda$ we unite

$$
0=\lambda\left(y_{\alpha}+y_{\beta}+y_{\gamma}+y_{\delta}+y_{s}\right)
$$

with

$$
\Phi=\mu_{\alpha} y_{\alpha}+\mu_{\beta} y_{\beta}+\mu_{\gamma} y_{\gamma}+\mu_{\delta} y_{\delta}+\mu_{\varepsilon} y_{s} \text {, }
$$

on dividing the result by $\mu_{\alpha}+\lambda$, and designating

we shall find

$$
\frac{\mu_{\tau}+\lambda}{\mu_{\alpha}+\lambda} \text { by } \nu_{\sigma}
$$

$$
\frac{\Phi}{\mu_{\alpha}+\lambda}=y_{\alpha}+v_{\beta} y_{\beta}+v_{\gamma} y_{\gamma}+v_{\delta} y_{\delta}+v_{\varepsilon} y_{\varepsilon} \text {. }
$$

Now in order that $\Phi$ may be different from

$$
\mu_{\alpha}\left(y_{\alpha}+y_{\beta}+y_{\gamma}+y_{\delta}+y_{\varepsilon}\right)
$$

$\lambda$ must admit of being determined so as to satisfy at lenst one equation of the form $\mu_{\nu}+\lambda=0$, without causing $\mu_{\alpha}+\lambda$ to vanish. If, therefore, we reflect that the system of equations on which $\Phi$ depends will remain unaltered if, while we substitute another imaginary root $i$ instead of $b$, we make certain substitutions among $y_{\beta}, y_{\gamma}, y_{\delta}, y_{s} ;$ and that consequently $\Phi$ may be deduced from

$$
\frac{\Phi}{\mu_{\alpha^{\prime}}+\lambda^{\prime}}=y_{\alpha}+v_{\beta^{\prime}} y_{\beta^{\prime}}+v_{\gamma^{\prime}} y_{\gamma^{\prime}}+v_{\gamma^{\prime}} y_{\delta^{\prime}}+v_{s^{\prime}} y_{s^{\prime}}
$$

$\nu_{v^{\prime}}$ being the same function of $i^{\prime}$ and $\lambda^{\prime}$ as $\nu_{v}$ is of, and $\lambda$, but $y_{v}$ being a different root from $y_{v}$ : we shall readily perceive that the coefficient of any one indifferently of the four roots $y_{\beta}, y_{\gamma}, y_{\delta}, y_{z}$ may be equated to zero, when the coefficient of $y_{\alpha}$ is equal to 1 .

Accordingly let us suppose that

$$
\nu_{s}=0 \text {, }
$$

and, on expressing $y_{a}, y_{\beta}, \ldots y_{\delta}$ in terms of $t$ and $u$, there will arise

$\frac{\Phi}{\mu_{\alpha}-\mu_{\varepsilon}}=\left(1+\imath \nu_{\beta}+\iota^{2} v_{\gamma}+\iota^{3} v_{\delta}\right) t+\left(1+\iota^{4} \nu_{\beta}+\iota^{3} v_{\gamma}+\iota^{2} v_{\delta}\right) u$.

This expression for $\frac{\Phi}{\mu_{\alpha}-\mu_{\varepsilon}}$ must in vanishing assume the form $0 t+0 u$. For $\frac{t}{u}$, which is not independent of $A_{2}^{\prime}, A_{5}^{\prime}$, 
cannot generally be equal to $-\frac{1+\imath^{4} \nu_{\beta}+\ldots}{1+1 v_{\beta}+\ldots}$. We must therefore have separately

$$
\begin{aligned}
& 1+\iota v_{\beta}+\iota^{2} y_{\gamma}+\iota^{3} y_{\delta}=0, \\
& 1+\iota^{4} y_{\beta}+\iota^{3} \nu_{\gamma}+\iota^{2} y_{\delta}=0 .
\end{aligned}
$$

If now we multiply the first of these equations by $i^{\zeta}$, and from the product subtract the second; we shall find

$$
\imath^{\zeta}-1+\left({ }^{\zeta+1}-b^{4}\right) \nu_{\beta}+\left(\imath^{\zeta+2}-b^{3}\right) \nu_{\gamma}+\left({ }^{\zeta+3}-\imath^{2}\right) \nu_{\delta}=0 \text { : }
$$

where $\zeta$ may have an unlimited number of different values assigned to it.

Hence, if we cause $v_{\gamma}$ to disappear by making $\zeta+2=3$, there will result

$$
v^{\beta}=v_{\delta}+a_{s}
$$

$a_{\text {, denoting }}-\frac{t-1}{t^{2}-t^{4}}$; and if $\zeta+1=4$, we shall have

$$
v_{\gamma}=a_{i} v_{\delta}+1 \text {, }
$$

$-\frac{i-\iota^{2}}{t-\iota^{3}}$ the coefficient of $v_{\delta}$ being evidently equal to $\frac{\iota}{t} a_{\imath}$.

Finally, on returning to the expression for $\frac{\Phi}{\mu_{\infty}+\nu}$ which involves $y_{a}, y_{\beta}, \ldots$, and making the requisite substitutions in it, we shall obtain

$$
\frac{\Phi}{\mu_{\alpha}-\mu_{\delta}}=y_{\alpha}+y_{\gamma}+a_{1} y_{\beta}+\left(y_{\beta}+y_{\delta}+a_{s} y_{\gamma}\right) \nu_{\delta} \text {; }
$$

which, if $\Phi=0$, will give, independently of $\nu_{\delta}$,

$$
\begin{aligned}
& \left.y_{\alpha}+y_{\gamma}+a_{\iota} y_{\beta}=0,\right\rceil \text {. . . . . . } \\
& y_{\beta}+y_{\delta}+a_{a} y_{\gamma}=0 \text {.J }
\end{aligned}
$$

And we see that the values of $p_{1}$ and $p_{2}$ which satisfy this pair of equations must be such as to fulfil the condition $\Phi=0$, or $\mu_{\alpha} y_{\alpha}+\mu_{\beta} y_{\beta}+\mu_{\gamma} y_{\gamma}+\mu_{\delta} y_{\delta}+\mu_{s} y_{s}=0$, and consequently to satisfy every one of the ten equations belonging to the same system.

7. We might now by means of these two equations, which involve $p_{1}, p_{2}, x_{\alpha}, x_{\beta}, x_{\gamma}, x_{\delta}$, and which are both of them of the first degree with respect to $p_{1}$ and $p_{2}$, express $p_{1}$ and $p_{2}$ as rational functions of $x_{\alpha}, x_{\beta}, x_{\gamma}, x_{\delta}$; and then, from discovering the number of different values which the expression for $p_{n}$ (either $p_{1}$ or $p_{2}$ ) would assume if the five indices $1,2,3,4,5$ 
were made to enter into it four at a time in every order of succession, determine the degree of the final equation $\chi_{n}\left(p_{n}, \mathbf{A}_{1}, \mathbf{A}_{2}, \ldots \mathbf{A}_{5}\right)=0 ; \chi_{n}$ representing a rational function. Certain properties of the roots of this equation would also become known. But we shall arrive far more rapidly at the same results, from considering the ten equations in question; the remaining eight of which are connected by a remarkable law with those already found.

8. It is obvious that we should have obtained a different pair of equations if, instead of supposing that $v_{s}=0$, we had equated another of the coefficients $\nu_{\beta}, v_{\gamma}, v_{\delta}, v_{\varepsilon}$, to zero. It will not, however, be necessary for us to retrace our steps in order to complete the system. From either of the equations (b.) we may discover all the rest. Thus if we take the first of them, and represent by

$$
y_{\alpha^{\prime}}+y_{\gamma^{\prime}}+a_{i} y_{\beta^{\prime}}=0
$$

what that equation will become if $i$ be substituted for , and the system (a.) remain unaltered; we shall see that, $i$ being different from $"$, there will arise a new equation belonging to the system. A difficulty here indeed presents itself. For if we write $\iota^{n} t^{\prime}$ for $t$ and $\iota^{4 n} u^{\prime}$ for $u$, it will be evident that we may obtain, corresponding to each of the four expressions $a_{t}, a_{t}, a_{t}, a_{t^{4}}$, five equations of the form in question. We appear therefore at first view to be conducted to twenty and not ten equations in the system. But an examination of the function $a_{i}$ will, as I proceed to show, point out the relation

$$
a_{i n}=a_{i 4 n}
$$

which includes these two conditions,

$$
a_{\imath}=a_{\iota}, \quad a_{\iota^{2}}=a_{t^{*}}
$$

9. Reverting to the expression for $a_{d}$, we see that

$$
\left(i^{2}-\imath^{4}\right) a_{t}=1-\imath
$$

Now $a$, considered generally as a rational function of may evidently be included under the form

where $c_{4}, c_{3}, \ldots c_{0}$ do not involve 1 .

$$
a_{t}=c_{4}+c_{3} t+c_{2} \iota^{2}+c_{1} \iota^{3}+c_{0} \iota^{4}
$$

Hence we find

$$
1-\imath=c_{1}-c_{3}+\left(c_{0}-c_{2}\right) \imath+\left(c_{4}-c_{1}\right) \iota^{2}+\left(c_{3}-c_{0}\right) \iota^{3}+\left(c_{2}-c_{4}\right) \iota^{4} ;
$$

which will be satisfied independently of , if

$$
\begin{gathered}
c_{1}-c_{3}=1, \quad c_{0}-c_{2}=-1, \\
c_{4}-c_{1}=0, \quad c_{3}-c_{0}=0, \quad c_{2}-c_{4}=0 .
\end{gathered}
$$

We thus perceive that 


$$
\begin{aligned}
a_{\imath} & =c_{1}\left(1+\imath^{2}+\iota^{3}\right)+c_{0}\left(\imath+\imath^{4}\right) \\
& =1+\imath^{2}+\imath^{3}+c_{0}\left(1+\imath+\imath^{2}+\imath^{3}+\iota^{4}\right)=-\left(\imath+\iota^{4}\right) .
\end{aligned}
$$

From which there will result, on writing $\iota^{n}$ instead of ,

$$
a_{i^{n}}=a_{4^{4}} \text {. }
$$

We might have arrived at the expression for $a_{t}$ from considering that $y_{a}+y_{\gamma}+a_{\jmath} y_{\beta}$, which expressed as a function of $t$ and $u$ would become $s^{4}\left(\imath^{4}+\imath+a_{t}\right) t+c\left(b+t^{4}+a_{t}\right) u$, must in vanishing assume the form $0 t+0 u$.

It appears then that $a_{i}$ has only two different values, $a_{t}$ and $a_{i}$. Hence all functions symmetric relatively to $a_{t}$ and $a_{i}$ will remain unaltered when we substitute any one of the three roots, $t^{2}, t^{3}, t^{4}$, instead of $t$.

And in accordance with this we find

$$
\begin{aligned}
& a_{\imath}+a_{i^{2}}=-\left(1+\imath+\iota^{2}+\iota^{3}+\iota^{4}\right)=-(-1), \\
& a_{s} \times a_{\iota^{2}}=(-1)^{2}\left(\iota+\iota^{4}\right)\left(\iota^{2}+\iota^{3}\right)=(-1)^{2}(-1) .
\end{aligned}
$$

$a_{t}$ and $a_{i^{2}}$ are in fact the roots of the equation

$$
a^{2}-a-1=0 \text {; }
$$

which solved as a quadratic equation will give

$$
a=\frac{1 \pm \sqrt{5}}{2}
$$

10. Another consequence of the properties of $a_{i}$ must here be pointed out.

Representing any one of the ten equations of the system by

$$
y_{\mathrm{a}}+y_{\mathrm{c}}+a_{\mathrm{i}^{n}} y_{\mathrm{b}}=0 \text {, }
$$

and observing that $a_{2} n$ is equal to $1-a_{1} 2 n$, we see that

$$
\begin{aligned}
y_{\mathrm{a}}+y_{\mathrm{c}}+a_{\mathrm{i} n} y_{\mathrm{b}} & =y_{\mathrm{a}}+y_{\mathrm{b}^{*}}+y_{\mathrm{c}}-a_{\mathrm{i}}{ }^{2 n} y_{\mathrm{b}} \\
& =-\left(y_{\mathrm{d}}+y_{\mathrm{e}}+a_{\mathrm{c}^{2 n}} y_{\mathrm{b}}\right) ;
\end{aligned}
$$

$a, b, \ldots$ e having, for greater simplicity, been introduced instead of the accented indices $\alpha^{\prime}, \beta^{\prime}, \ldots \varepsilon^{\prime}$.

Thus it appears that $p_{1}$ and $p_{2}$ cannot be determined by means of any two equations expressible by

$$
\left.\begin{array}{c}
y_{\mathrm{a}}+y_{\mathrm{c}}+a_{,} y_{\mathrm{b}}=0, \\
y_{\mathrm{d}}+y_{\mathrm{e}}+a^{2 n} y_{\mathrm{b}}=0,
\end{array}\right\} . . . .
$$

which, although seeningly independent of each other, are in reality reducible to a single equation.

11. In discussing equations of the class (c.), the following definition will be found useful :-

Of the two functions $y_{\mathrm{a}}+y_{\mathrm{c}}+a_{\mathrm{b}} y_{\mathrm{b}}, y_{\mathrm{d}}+y_{\mathrm{e}}+a_{\mathrm{c}}{ }^{n} y_{\mathrm{b}}$, which are of the same form, and which taken together include 
all the roots of the equation for $y, y_{\mathrm{b}}$ remaining fixed; the one will be said to be the complement of the other: and either of these functions with the letter $\mathfrak{c}$ prefixed to it as a characteristic will express symbolically the complement of that function.

12. It may now be seen, either from multiplying $y_{\alpha}, y_{\beta}, \ldots y_{3}$ successively by $a_{\text {, }}$ or from the law of the indices in the equations (b.), that the ten equations,

$$
\begin{aligned}
& y_{\varepsilon}+y_{\beta}-\left(\imath+\imath^{4}\right) y_{\alpha}=0, \\
& y_{\alpha}+y_{\gamma}-\left(\iota+\iota^{4}\right) y_{\beta}=0 \text {, } \\
& y_{\beta}+y_{\delta}-\left(1+{ }^{4}\right) y_{\gamma}=0 \text {, } \\
& y_{\gamma}+y_{\varepsilon}-\left(t+\imath^{4}\right) y_{\delta}=0 \text {, } \\
& y_{\delta}+y_{\alpha}-\left(1+1^{4}\right) y_{\varepsilon}=0, \\
& \text { c }\left(y_{\varepsilon}+y_{\beta}-\left(\iota^{2}+\iota^{3}\right) y_{\alpha}\right)=0 \text {, } \\
& c\left(y_{\alpha}+y_{\gamma}-\left(\imath^{2}+\imath^{3}\right) y_{\beta}\right)=0 \text {, } \\
& c\left(y_{\beta}+y_{\delta}-\left(\imath^{2}+\imath^{3}\right) y_{\gamma}\right)=0 \\
& \mathfrak{c}\left(y_{\gamma}+y_{\imath}-\left(\iota^{2}+\iota^{s}\right) y_{\delta}\right)=0 \text {, } \\
& c\left(y_{\delta}+y_{\alpha}-\left(\iota^{2}+\iota^{3}\right) y_{\varepsilon}\right)=0, j
\end{aligned}
$$

where the indices in each vertical column follow the same order of succession as in the cycle

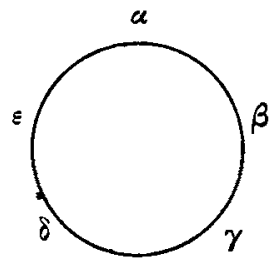

cannot, whilst $\alpha, \beta, \ldots$ remain unaltered, condnct to more than one set of values for $p_{1}$ and $p_{2}$.

But before discussing the equations (d.) and (e.), I proceed to consider properties of functions in which the elements are supposed to change places among themselves.

\section{Section II.}

13. Let $\mathrm{X}$ represent a function of $\boldsymbol{n}$ independent quanti-

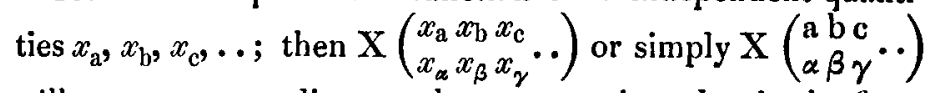
will express, according to a known notation, that in the function $\mathrm{X}$ the quantities $x_{a}, x_{\mathrm{b}}, x_{\mathrm{c}}, \ldots$ have been changed into 


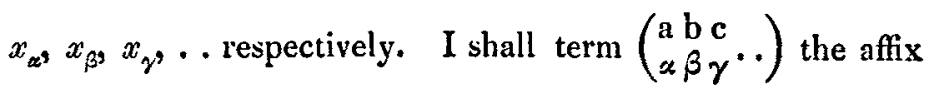
of substitution.

14. The number of different values which $X$ can receive when we change the order of the elements on which it depends cannot exceed the product $1.2 .3 \ldots n$; but the affix of substitution will admit of $1.2 .3 \ldots n \times 1.2 .3 \ldots n$ differently derived expressions.

Thus if we denote by $\Lambda_{1}, \Lambda_{2}, \ldots \Lambda_{1,2,3 \ldots n}$ the different forms or states which the five indices $(1,2,3,4,5)$ are capable of assuming from the several changes of arrangement to which they are supposed to be subjected, the values of $X$ may all of them be expressed by

$$
\mathrm{X}\left(\begin{array}{l}
\Lambda_{1} \\
\Lambda_{1}
\end{array}\right), \mathrm{X}\left(\begin{array}{l}
\Lambda_{1} \\
\Lambda_{2}
\end{array}\right), \mathrm{X}\left(\begin{array}{l}
\Lambda_{1} \\
\Lambda_{3}
\end{array}\right), \ldots \mathrm{X}\left(\begin{array}{l}
\Lambda_{1} \\
\Lambda_{v}
\end{array}\right)
$$

$\nu$ denoting the product $1.2 .3 \ldots n$; but in this system we may successively substitute $\Lambda_{2}, \Lambda_{3}, \ldots \Lambda_{\nu}$ instead of $\Lambda_{1}$ : whence will result $(\nu-1)$ other systems, each of them consisting of $\nu$ terms.

15. Suppose $X$ to be such that the number of different values of which it is susceptible shall be less than $v$.

Here certain terms in the system

$$
\mathrm{X}\left(\begin{array}{l}
\Lambda_{1} \\
\Lambda_{1}
\end{array}\right), \mathrm{X}\left(\begin{array}{l}
\Lambda_{1} \\
\Lambda_{2}
\end{array}\right), \ldots \mathrm{X}\left(\begin{array}{l}
\Lambda_{1} \\
\Lambda_{v}
\end{array}\right)
$$

must be equal to each other.

Let therefore

$$
\mathrm{X}\left(\begin{array}{l}
\Lambda_{1} \\
\Lambda_{1}
\end{array}\right)=\mathrm{X}\left(\begin{array}{l}
\Lambda_{1} \\
\Lambda_{2}
\end{array}\right)=\ldots=\mathrm{X}\left(\begin{array}{l}
\Lambda_{1} \\
\Lambda_{\mu}
\end{array}\right) .
$$

On submitting each of these $\mu$ expression to the substitution denoted by $\left(\begin{array}{l}\Lambda_{1} \\ \Lambda_{\mu+1}\end{array}\right)$, and observing that instead of an expression of the form

$$
\mathrm{X}\left(\begin{array}{l}
\Lambda_{1} \\
\Lambda_{\tau}
\end{array}\right)\left(\begin{array}{l}
\Lambda_{1} \\
\Lambda_{\mu+1}
\end{array}\right)
$$

where $\mathrm{X}$ has been subjected to two successive substitutions, we may write

$$
\mathrm{X}\left(\begin{array}{c}
\Lambda_{1} \\
\Lambda_{v}
\end{array}\right)
$$

we shall have no difficulty in perceiving that the new set of equal quantities which will arise may be represented by

$$
\mathrm{X}\left(\begin{array}{l}
\Lambda_{1} \\
\Lambda_{\mu+1}
\end{array}\right)=\mathrm{X}\left(\begin{array}{l}
\Lambda_{1} \\
\Lambda_{\mu+2}
\end{array}\right)=\ldots=\mathrm{X}\left(\begin{array}{l}
\Lambda_{1} \\
\Lambda_{2 \mu}
\end{array}\right):
$$


which quantities are different from the former, but equal to them in number.

If we operate in the same manner with $\left(\begin{array}{l}\Lambda_{1} \\ \Lambda_{2 \mu+1}\end{array}\right),\left(\begin{array}{l}\Lambda_{1} \\ \Lambda_{3 \mu+1}\end{array}\right)$, .. $\left(\begin{array}{l}\Lambda_{1} \\ \Lambda_{(\omega-1) \mu+1}\end{array}\right)$ until we have exhausted all the substitutions, we shall find that the $y$ values of $X$ will be separated into $w$ groups composed each of them of $\mu$ terms.

Hence the number of different values which a function of $n$ quantities may receive from all the possible substitutions of these quantities among themselves, is necessarily a submultiple of the product $1.2 .3 \ldots n$, as is well known.

16. If $\mathrm{X}$ be affected by a series of contiguous* substitutions,

we shall have generally

$$
\left(\begin{array}{l}
\Lambda_{1} \\
\Lambda_{2}
\end{array}\right),\left(\begin{array}{l}
\Lambda_{2} \\
\Lambda_{3}
\end{array}\right),\left(\begin{array}{l}
\Lambda_{3} \\
\Lambda_{4}
\end{array}\right), \ldots\left(\begin{array}{l}
\Lambda_{\mu-1} \\
\Lambda_{\mu}
\end{array}\right)
$$

$$
\mathrm{X}\left(\begin{array}{l}
\Lambda_{1} \\
\Lambda_{2}
\end{array}\right)\left(\begin{array}{l}
\Lambda_{2} \\
\Lambda_{3}
\end{array}\right)\left(\begin{array}{l}
\Lambda_{3} \\
\Lambda_{4}
\end{array}\right) \cdot .\left(\begin{array}{l}
\Lambda_{\mu-1} \\
\Lambda_{\mu}
\end{array}\right)=\mathrm{X}\left(\begin{array}{l}
\Lambda_{1} \\
\Lambda_{\mu}
\end{array}\right)
$$

This is evident.

17. Let us now consider

$$
\mathrm{X}\left(\begin{array}{c}
\Lambda_{1} \\
\Lambda_{v}
\end{array}\right)\left(\begin{array}{c}
\Lambda_{1} \\
\Lambda_{\nu}
\end{array}\right)\left(\begin{array}{c}
\Lambda_{1} \\
\Lambda_{\nu}
\end{array}\right)
$$

where the same substitution is supposed to be applied any number of times in succession to the function $\mathrm{X}$.

It is obvious that a limited number, $p$, of such operations must bring us to an expression equal to $X$; and that all the expressions previously obtained will then reappear in a periodical manner.

If, in effect, we denote by $\mathrm{X}\left(\begin{array}{c}\Lambda_{1} \\ \Lambda_{\nu}\end{array}\right)^{r}$ the value of $\mathrm{X}$ which will arise when the substitution designated by $\left(\begin{array}{c}\Lambda_{1} \\ \Lambda_{v}\end{array}\right)$ has been applied $r$ times; we shall have

$$
\mathrm{X}\left(\begin{array}{c}
\Lambda_{1} \\
\Lambda_{\nu}
\end{array}\right)^{0}, \mathrm{X}\left(\begin{array}{c}
\Lambda_{1} \\
\Lambda_{\nu}
\end{array}\right)^{1}, \mathrm{X}\left(\begin{array}{c}
\Lambda_{1} \\
\Lambda_{\nu}
\end{array}\right)^{2}, \ldots \mathrm{X}\left(\begin{array}{c}
\Lambda_{1} \\
\Lambda_{\nu}
\end{array}\right)^{p-1}
$$

after which we shall come to the term $\mathrm{X}\left(\begin{array}{c}\Lambda^{\mathrm{I}} \\ \Lambda_{v}\end{array}\right)^{p}$ which, by hypothesis, is equal to $\mathrm{X}$ or $\mathrm{X}\left(\begin{array}{c}\Lambda_{1} \\ \Lambda_{v}\end{array}\right)^{0}$; and consequently if

- A term made use of in connexion with substitutions by M. Cauchy, to whom we are indebted for the results in (17.) and (18.), as well as for the theorem given above in (16.). 
we continue to operate with $\left(\begin{array}{l}\Lambda_{1} \\ \Lambda_{v}\end{array}\right)$ we shall merely reproduce the same series of $p$ terms disposed in the same order as before.

Thus we shall obtain, in the form of an equation,

$$
\mathrm{X}\left(\begin{array}{c}
\Lambda_{1} \\
\Lambda_{\nu}
\end{array}\right)^{a p+r}=\mathrm{X}\left(\begin{array}{c}
\Lambda_{1} \\
\Lambda_{\nu}
\end{array}\right)^{r}
$$

$a$ and $r$ representing any integers, zero included. What is termed the degree of $\left(\begin{array}{l}\Lambda_{1} \\ \Lambda_{v}\end{array}\right)$ is indicated by $p$, which is, as might easily be shown, equal to the number of the indices $\alpha, \beta, \gamma, \ldots$ contained in the affix when reduced to its most simple expression*.

18. An important theorem on the decomposition of substitutions here presents itself.

Observing that if

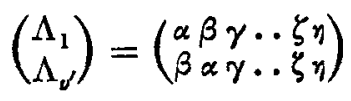

we shall have

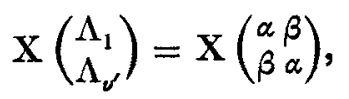

where $\left(\begin{array}{l}\alpha \beta \\ \beta\end{array}\right)$ indicates an interchange or transposition of the elements $\alpha$ and $\beta$; and that if

we shall have

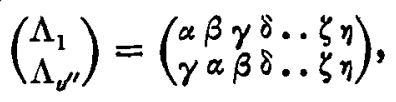

$$
\begin{aligned}
\mathrm{X}\left(\begin{array}{c}
\Lambda_{1} \\
\Lambda_{v^{\prime \prime}}
\end{array}\right) & =\mathrm{X}\left(\begin{array}{l}
\alpha \beta \gamma \\
\gamma \alpha \beta
\end{array}\right) \\
& =\mathrm{X}\left(\begin{array}{l}
\alpha \beta \gamma \\
\beta \alpha \gamma
\end{array}\right)\left(\begin{array}{l}
\beta \alpha \gamma \\
\gamma \alpha \beta
\end{array}\right)=\mathrm{X}\left(\begin{array}{l}
\alpha \beta \\
\beta \alpha
\end{array}\right)\left(\begin{array}{l}
\beta \gamma \\
\gamma \beta
\end{array}\right), \quad \text { (see 16.) }
\end{aligned}
$$

the operation denoted by $\left(\begin{array}{l}\Lambda_{1} \\ \Lambda_{v^{\prime \prime}}\end{array}\right)$ being in this case equivalent to the two interchanges $\left(\begin{array}{l}\alpha \beta \\ \beta\end{array}\right)$ and $\left(\begin{array}{l}\beta \gamma \\ \gamma \beta\end{array}\right)$ taken in succession; we are at once led to infer that every substitution may be represented by a succession of interchanges.

And in effect if

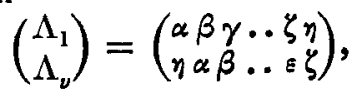

* Examples of such a reduction will appear in (18). 
there will arise

$$
\mathrm{X}\left(\begin{array}{c}
\Lambda_{1} \\
\Lambda_{v}
\end{array}\right)=\mathrm{X}\left(\begin{array}{c}
\alpha \beta \\
\beta \alpha
\end{array}\right)\left(\begin{array}{l}
\beta \gamma \\
\gamma \beta
\end{array}\right) \cdots\left(\begin{array}{l}
\zeta \eta \\
\eta \zeta
\end{array}\right)
$$

as will be evident on reflecting that

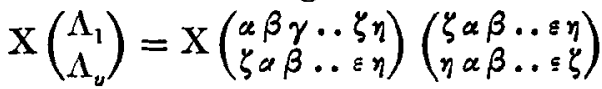

$$
\begin{aligned}
& =\mathrm{X}\left(\begin{array}{cccc}
\alpha & \beta & \ddots & \zeta \\
\zeta & \beta & \ldots & \ldots
\end{array}\right)\left(\begin{array}{l}
\zeta \eta \\
\eta
\end{array}\right),
\end{aligned}
$$

or that every substitution of the $n$th degree, $n$ being any number, may be represented by a substitution of the $(n-1)$ th degree followed by an interchange.

19. I proceed to consider some properties of the function

$$
\mathrm{X}\left(\begin{array}{c}
\alpha \beta \\
\beta \alpha
\end{array}\right)\left(\begin{array}{c}
\gamma \delta \\
\delta \gamma
\end{array}\right) \ldots
$$

which in accordance with the meaning usually attached to the symbol (..), I shall express by

$$
\mathrm{X}(\alpha \beta)\left(\gamma_{. .}^{\delta}\right) \cdots
$$

(ab) thus denoting the same thing as $\left(\begin{array}{l}a b \\ b a\end{array}\right)$.

20. Beginning with the function $X(\alpha \beta)$, it will at once be seen that

$$
X(\alpha \beta)=X(\beta . .)
$$

$(\alpha \beta)$ and $(\beta \alpha)$ being equally expressive of an interchange of the elements $\alpha$ and $\beta$.

21. Passing to the function $X(\alpha \beta)(\gamma .$.$) , we see that, if$ $\alpha, \beta, \gamma, \delta$ be unequal, we must have

$$
X(\alpha \beta)(\gamma \delta)=X(\gamma \delta)(\alpha \beta) . . . .
$$

For the interchanges being, according to this hypothesis, independent of each other, it must be indifferent in what order they are taken.

But if the function in question were of the form $\mathrm{X}(\alpha \beta)(\beta \gamma)$, in which the element $\beta$ is common to the interchanges, we should, exclusively of particular cases, alter the value of the function by inverting the order of the interchanges.

'Thus if

$$
\mathrm{X}=\Psi(\alpha, \beta, \gamma) \text {, }
$$

we shall have

$$
\begin{aligned}
& \mathrm{X}(\alpha \beta)(\beta \gamma)=\Psi(\gamma, \alpha, \beta), \\
& \mathrm{X}(\ddot{\beta}, \gamma)\left(\alpha \beta^{\prime}\right)=\Psi(\alpha, \gamma, \beta)\left(\alpha \beta^{\prime}\right),
\end{aligned}
$$

and

$\beta^{\prime}$ being arbitrary.

Now, if $\Psi(\gamma, \alpha, \beta)=\Psi(\alpha, \gamma, \beta)\left(\alpha \beta^{\prime}\right)$, we must take $\beta^{\prime}=\gamma$. There will consequently result

$$
\mathrm{X}(\alpha \beta)(\beta, ., \gamma)=\mathrm{X}(\beta, \gamma)(\alpha, \gamma) ; . .
$$


in the second member of which equation the element $\gamma$, and not $\beta$, is common to the interchanges. $\alpha, \beta, \gamma$ are supposed to be unequal. In $X(\alpha \beta)(\alpha \beta)$ an inversion of the interchanges can take place without disturbing the value of that function.

Further, it is clear from the equation $(\beta$. ) that

$$
\mathrm{X}(\beta . \gamma)(\alpha, \gamma)=\mathrm{X}(\alpha, \gamma)(\alpha \beta) \text {, }
$$

and

$$
\mathrm{X}(\alpha, \gamma)(\alpha \beta)=\mathrm{X}(\alpha \beta)(\beta, \gamma)
$$

so that we shall have the three equal expressions

$$
\mathrm{X}(\alpha \beta)(\beta \gamma), \mathrm{X}(\beta, \gamma)(\alpha \gamma), \mathrm{X}(\alpha, \gamma)(\alpha \beta) \text {; }
$$

which, if we continue to apply the equation $(\beta$.$) , will reappear$ periodically.

22. With respect to $\mathrm{X}(\alpha \beta)(\underline{\beta} \hat{\beta})(\xi \xi)$, on denoting it by $\mathrm{Y}$ we shall find, since $\mathrm{X}_{u}(\epsilon \zeta)^{2}=\mathrm{X}_{u}$,

$$
\mathrm{X}(\alpha \beta)\left(\gamma_{.}^{\delta}\right)=\mathrm{Y}(\varepsilon, \zeta) \text {. }
$$

If therefore we substitute $* \mathrm{X}\left(\underline{\gamma}^{\delta}\right)\left(\alpha \beta^{\prime}\right)$ instead of $\mathrm{X}(\alpha \beta)\left(\gamma^{\delta}\right)$, and affect with $(\varepsilon, \zeta)$ both members of the equation which will thence arise, we shall have

$$
\mathrm{X}\left(\gamma_{. .}^{\delta}\right)\left(\alpha \beta^{\prime}\right)(\xi . \zeta)=\mathrm{Y}=\mathrm{X}(\alpha \beta)\left(\gamma_{.}^{\delta}\right)(\xi \zeta) ;
$$

that is, we can operate with the first and second of the interchanges as if the third did not exist. In like manner it might be shown that in operating with the second and third we may neglect the first.

And analogous results will be obtainable whatever may be the number of the interchanges with which $\mathrm{X}$ is affected.

\section{Section III.}

23. Returning now to the equations (d.), and designating them, in the order in which they occur, by $f_{\alpha}=0, f_{\beta}=0$, $\ldots f_{i}=0$, we find, on inspection,

$$
\begin{aligned}
& f_{\alpha}(\alpha \beta)=f_{\beta}(\varepsilon \gamma), \\
& f_{\gamma}(\alpha \beta)=f_{\varepsilon}(\varepsilon \gamma) .
\end{aligned}
$$

We also find, as might have been foreseen,

$$
\begin{aligned}
& f_{\beta}(\alpha \beta)=f_{\alpha}(\varepsilon \gamma), \\
& f_{1}(\alpha \beta)=f_{\gamma}(\varepsilon, ?) .
\end{aligned}
$$

And it will have been observed that the two functions in-

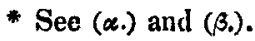


volved in any of these four equations are either $f_{\alpha}, f_{\beta}$, or $f_{s}, f_{\gamma}$; the indices being in the former case equal to the elements of the given affix $(\alpha \beta)$; and in the latter to those of $(q)$. Further, we obtain

$$
f_{\delta}(\alpha \beta)=f_{\delta}(\varepsilon \gamma)
$$

which involves the single function $f_{\delta}$, the index of which, $\delta$, does not enter into either $(\alpha \underline{\beta})$ or $(\varepsilon, \gamma)$.

We are thus conducted to the equation (20.),

$$
f_{\mathrm{a}}(\alpha \beta)=f_{\mathrm{b}}\left(\gamma_{. .} \boldsymbol{\beta}\right)
$$

where $a$ and $b$ are such as, abstractedly of the order in which they are arranged, to be restricted to the three sets of values

$$
\left.\left.\left.\begin{array}{l}
\alpha \\
\beta
\end{array}\right\}, \begin{array}{l}
\gamma \\
\varepsilon
\end{array}\right\}, \quad \begin{array}{l}
\delta \\
\delta
\end{array}\right\}
$$

24. When $\alpha$ instead of occurring among the elements to be interchanged remains fixed, we have

$\mathrm{b}$ and $\mathrm{e}$ here depending on

$$
f_{b}\left(\beta_{. \varepsilon}\right)=f_{\mathrm{e}}\left(\gamma_{.} \delta\right), \quad \cdot \quad \cdot \cdot \cdot
$$

$$
\left.\left.\left.\begin{array}{l}
\beta \\
\varepsilon
\end{array}\right\}, \begin{array}{l}
\gamma \\
\delta
\end{array}\right\}, \begin{array}{l}
\alpha \\
\alpha
\end{array}\right\} .
$$

This theorem may either be derived from the preceding one, or obtained directly from the equations (d.).

25. Finally, we obtain

$$
f_{\mathrm{b}}\left(\beta_{. \gamma}\right)(\delta \varepsilon)=\left(c f_{\mathrm{c}}\right)\left(\beta_{\mathrm{.}}\right)_{n}
$$

where $\left(\beta_{.}\right)_{n}$ must coincide either with $\left(\beta_{. .}\right)$or with $\left(\gamma_{. \delta}^{\delta}\right)$, the complementary interchange relatively to $f_{\alpha}$. With respect to $b$ and $c$, if we take $b$ successively equal to

$$
\alpha, \beta, \gamma, \delta, \varepsilon \text {, }
$$

the corresponding values of $\mathrm{c}$ will be, if $\left(\beta_{. .}\right)_{n}=\left(\beta_{. .}\right)$,

but if $\left(\beta_{.}\right)_{n}=\left(\gamma_{. .}^{\delta}\right)$, they will be

$$
\alpha, \gamma, \varepsilon, \beta, \delta \text {; }
$$

$$
\alpha, \delta, \beta, \varepsilon, \gamma
$$

the successive values of $\mathbf{c}$ being in each case arranged at equal intervals in the cycle formed with the indices of the equations (d.) or (e.) taken in order.

And a similar theorem will exist for $f_{\mathrm{b}}\left(\beta_{. .} \delta\right)\left(\gamma_{. .}\right)$.

\section{Section IV.}

26. The final equation on which $p_{1}, p_{2}, p_{3}$ depend, is of the $(1$.3.4)th degree. This result, which will have been fore- 
seen independently of the form of that equation, or of the nature of its roots*, may be deduced anew, in conjunction with some very remarkable properties of the routs in question, from the theorems given in the last section.

Designating by $\mathrm{P}$ one of the quantities $p_{1}, p_{2}$, we perceive from the equation (f.), or rather from

$$
f_{\mathrm{a}^{\prime}}\left(\alpha \beta^{\prime}\right)=f_{\mathrm{b}^{\prime}}\left(\gamma_{.}^{\prime} \xi^{\prime}\right)
$$

(in which $\beta^{\prime}$ may be supposed to be equal to any one of the four indices, $\beta, \gamma, \delta, \varepsilon$; and $\gamma^{\prime}$, $\varepsilon^{\prime}$ to be each of them different from $\alpha$ ), that the expression for $P$, considered as a function of $x_{\alpha}, x_{\beta}, \ldots x_{s}$, will be such as to assume all its values whilst one of the roots $x_{\alpha}$ remains fixed; and $x_{\beta}, x_{\gamma}, x_{\delta}, x_{\varepsilon}$ undergo among themselves all the different changes of arrangement to which they can be subjected. It appears therefore already that the equation on which $P$ depends cannot rise above the $(1.2 .3 .4)$ th degree.

Again, it is evident from the next theorem (g.) that of the four pairs of equations to which all those which include $f_{*}$, or $y_{s}+y_{\beta}-\left(t+\imath^{4}\right) y_{\alpha}$, are reducible,

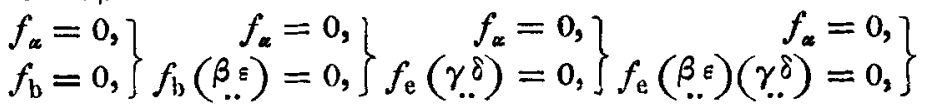

the first will furnish the same expression for $\mathbf{P}$ as the fourth; and the second as the third.

And since $f_{\alpha}$ will not, whilst $\alpha$ remains fixed, admit of more than $\frac{4 \times 3}{1 \times 2}$ different expressions, the number of different values which may be assigned to $\mathrm{P}$ cannot exceed $\frac{4}{2} \times \frac{4 \times 3}{1 \times 2}$.

$P$ therefore will depend on an equation of the 12th degree, or rather on an equation of the form

$$
\left(\mathrm{P}^{12}+\mathrm{B}_{1} \mathrm{P}^{11}+\mathrm{B}_{2} \mathrm{P}^{10}+\ldots+\mathrm{B}_{12}\right)^{10}=0 ;
$$

in which $\mathrm{B}_{1}, \mathrm{~B}_{2}, \ldots \mathrm{B}_{12}$ are symmetric relatively to $x_{1}, x_{2}, \ldots x_{5}$, and may consequently, as is well known, be expressed as rational functions of $A_{1}, A_{2}, \ldots A_{5}$, the coefficients of the original equation.

27. To obtain the roots of this equation in terms of $x_{1}, x_{2}$, $\ldots x_{5}$, let us suppose that

$$
\alpha=1 ;
$$

* From considering that the equations

$$
A_{1}^{\prime}=0, \quad A_{3}^{\prime}=0, \quad A_{4}^{\prime}-\frac{1}{5} A_{2}^{\prime}=0
$$

are of the first, third, and fourth degrees relatively to $p_{1}, p_{2,} p_{3}$. 
we shall then, by making the following substitutions with $\beta$ and $\varepsilon$,

$$
\begin{aligned}
& \left(\begin{array}{ll}
\beta & \varepsilon \\
2 & 5
\end{array}\right), \quad \quad\left(\begin{array}{ll}
\beta & \varepsilon \\
3 & 4
\end{array}\right), \\
& \left(\begin{array}{ll}
\beta & \varepsilon \\
3 & 5
\end{array}\right), \quad \quad\left(\begin{array}{ll}
\beta & \varepsilon \\
2 & 4
\end{array}\right), \\
& \left(\begin{array}{ll}
\beta & \varepsilon \\
4 & 5
\end{array}\right), \quad \quad\left(\begin{array}{ll}
\beta & \varepsilon \\
2 & 3
\end{array}\right),
\end{aligned}
$$

find the six expressions of which $f_{1}$ is susceptible,

$$
\begin{aligned}
& y_{2}+y_{5}-\left(1+\imath^{4}\right) y_{1}, \quad y_{3}+y_{4}-\left(1+\imath^{4}\right) y_{1} \text {, } \\
& y_{3}+y_{5}-\left(1+\imath^{4}\right) y_{1}, \quad y_{2}+y_{4}-\left(1+1^{4}\right) y_{1} \text {, } \\
& y_{4}+y_{5}-\left(1+t^{4}\right) y_{1}, \quad y_{2}+y_{3}-\left(1+t^{4}\right) y_{1} \text {; }
\end{aligned}
$$

or, if we designate the expressions in the first of these columns by $i_{1}, k_{1}, l_{1}$, respectively,

$$
\begin{array}{ll}
i_{1}, & \mathrm{c} i_{1}, \\
k_{1}, & \mathrm{c} k_{1}, \\
l_{1}, & \mathrm{c} l_{1} ;
\end{array}
$$

each expression in one column being the complement of the corresponding expression in the other.

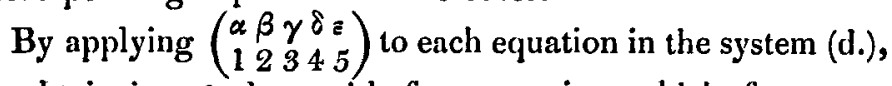
we obtain $i_{1}=0$ along with four equations which, from ana$\operatorname{logy}$, we shall indicate by $i_{2}=0, i_{3}=0, i_{4}=0, i_{5}=0$, taken in order.

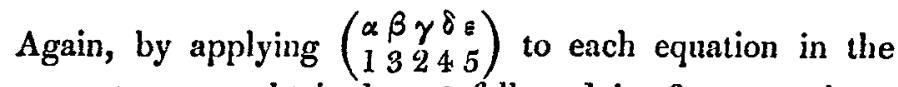
same system, we obtain $k_{1}=0$ followed by four equations, which abstractedly of the order in which they present themselves, will be indicated by $k_{2}=0, k_{3}=0, k_{4}=0, k_{5}=0$; the index of $k$ being made to correspond with the index of the term multiplied by $a_{i}$.

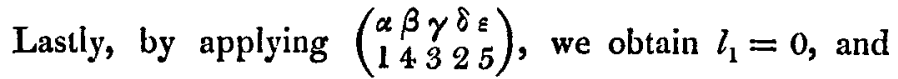
$l_{2}=0, l_{3}=0, l_{4}=0, l_{5}=0$.

Now, from the theorem (h.) there will result

$$
\begin{aligned}
& i_{\mathrm{b}^{\prime}}(23)\left(\begin{array}{l}
4.5) \\
. . .
\end{array}\right)\left(c i_{\mathrm{c}^{\prime}}\right)(2.5)_{n} \text {, } \\
& k_{\mathrm{b}^{\prime \prime}}\left(\begin{array}{c}
32 \\
. .1
\end{array}\right)(15)=\left(\mathrm{c} k_{\mathrm{c}^{\prime \prime}}\right)\left({ }^{35}\right)_{n} \text {, } \\
& l_{\mathrm{b}^{\prime \prime \prime}}(43)(25)=\left(c l_{\mathrm{c}^{\prime \prime \prime}}\right)(4.5)_{n} \text {, }
\end{aligned}
$$

the second members of which will, if $b^{\prime}=b^{\prime \prime}=b^{\prime \prime \prime}=1$, reduce themselves to $\mathrm{c} i_{1}, \mathfrak{c} k_{1}, \mathfrak{c} l_{1}$.

If then we observe that generally (10.)

$$
c f_{\tau}=-f_{\tau}^{\prime} \text {; }
$$


$f_{\tau}^{\prime}$ denoting what $f_{\tau}$ becomes when $a_{1}$ is changed into $a_{\tau^{2}}$; we shall readily perceive that the six groups of equations on which, as we have seen, all the different expressions for $P$ depend will be reducible to the three following groups,

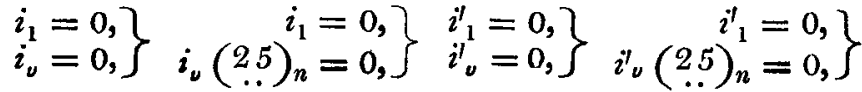

$$
\begin{aligned}
& \left.\left.\left.\left.\begin{array}{r}
k_{1}=0, \\
k_{v}=0,
\end{array}\right\} \quad \begin{array}{r}
k_{1}=0, \\
k_{\nu}(35)_{n}=0,
\end{array}\right\} \quad \begin{array}{l}
k_{1}^{\prime}=0, \\
k_{v}^{\prime}=0,
\end{array}\right\} \quad \begin{array}{r}
k_{v}(35)_{n}=0, \\
k_{v}=0,
\end{array}\right\} \\
& \left.\left.\left.\left.\begin{array}{l}
l_{1}=0, \\
l_{v}=0,
\end{array}\right\} \quad \begin{array}{r}
l_{1}=0, \\
l_{v}(4.5)_{n}=0,
\end{array}\right\} \begin{array}{l}
l_{1}^{\prime}=0, \\
l_{v}=0,
\end{array}\right\} \quad \begin{array}{r}
l_{1}^{\prime}=0, \\
l_{v}(4.5)_{n}=0,
\end{array}\right\}
\end{aligned}
$$

$(25)_{n},(35)_{n},(45)_{n}$ may denote either the three inter-

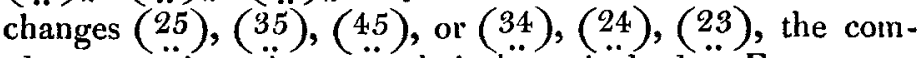
plementary interchanges relatively to $i_{1}, k_{1}, l_{1}$. For greater uniformity the same index $v$ has been retained throughout the groups; the expression for $\mathrm{P}$ being in every case unaffected in value ly writing $2,3,4,5$ successively instead of $U$.

28. But for our purpose it will not be necessary from each pair of these equations actually to find an expression for $\mathbf{P}$ in terms of $x_{1}, x_{2}, \ldots x_{5}$. In effect, if we denote by $\mathrm{P}_{f(\mathrm{ab})(\mathrm{cd}) \text {.. }}$ that value of $\mathbf{P}$ which is derived from the pair of equations $f_{\alpha}(\mathrm{ab})(\mathrm{cd}) \ldots=0, f_{\nu}(\mathrm{ab})(\mathrm{cd}) \ldots=0$; the twelve values

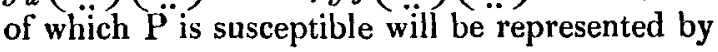

$$
\left.\begin{array}{llll}
\mathrm{P}_{i}, & \mathrm{P}_{i(25)_{n}}, & \mathrm{P}_{i^{\prime}}, & \mathrm{P}_{i^{\prime}(25)_{n}}, \\
\mathrm{P}_{k}, & \mathrm{P}_{k(35)_{n}}, & \mathrm{P}_{k^{\prime}}, & \mathrm{P}_{k^{\prime}(35)_{n}}, \\
\mathrm{P}_{l}, & \mathrm{P}_{l\left({ }_{(4 .}\right)_{n}}, & \mathrm{P}_{l^{\prime}}, & \mathrm{P}_{l^{\prime}\left({ }_{.}(5)_{n}\right.}:
\end{array}\right\} . . .
$$

so that without proceeding any further we may perceive that the roots of the equation $\mathrm{P}^{12}+\mathrm{B}_{1} \mathrm{P}^{11}+\ldots=0$ must be such as to admit of being distributed into three groups which are related to each other in a very remarkable manner; the second pair of roots in each group being derivable from the first pair by merely introducing $a_{b^{2}}$ instead of $a_{i}$. Indeed the groups themselves may all of them be derived from

$$
\mathrm{P}_{f}, \quad \mathrm{P}_{f\left(\beta_{.} \xi\right)_{n}}, \quad \mathrm{P}_{f^{\prime}}, \mathrm{P}_{f^{\prime}\left(\beta_{. .}\right)_{n}},
$$

which will represent four roots of the equation for $P$.

\section{Section V.}

29. At first view it might be imagined that, if

$$
\mathrm{V}_{\mathrm{F}}=\mathrm{P}_{f}+\mathrm{P}_{f\left(\beta_{.}\right)_{n}}+\mathrm{P}_{f^{\prime}}+\mathrm{P}_{f^{\prime}\left(\beta_{. .8}\right)_{n}},
$$

Phil. Mag. S. 3. No. 176. Suppl. Vol. 26. 
the equation for $V_{F}$ would not rise above the third degree. But although the eight functions,

\begin{tabular}{|c|c|}
\hline$V_{\mathrm{F}}$ & $V_{F^{\prime}}$ \\
\hline $\mathrm{V}_{\mathrm{F}\left(\beta_{n}\right)}$, & $\mathrm{V}_{\mathrm{F}^{\prime}\left(\beta_{. .}\right)}$, \\
\hline $\mathrm{V}_{\mathrm{F}\left(\gamma^{\prime}\right)}$, & $\mathrm{V}_{\mathrm{F}^{\prime}\left(y^{\prime}\right)}$ ? \\
\hline
\end{tabular}

$\mathrm{V}_{\mathrm{F}^{\prime}}$ denoting what $\mathrm{V}_{\mathrm{F}}$ becomes when $f$ is changed into $f^{\prime}$, will be necessarily equal to each other: and consequently if $f$ be changed successively into $i, k, l$, and $\mathrm{F}$ into $\mathrm{I}, \mathrm{K}, \mathrm{L}$, there will arise, the index 1 remaining fixed, eight functions equal to $V_{I}$; eight to $V_{K}$; and eight to $V_{L}$; we must not conclude that $V_{F(1 \beta)}$, in which the index 1 is supposed not to be fixed, will be capable of coinciding with one of the three functions $V_{I}, V_{K}, V_{L}$. It is true that all the roots of the equation $\mathrm{P}^{12}+\mathrm{B}_{1} \mathrm{P}^{11}+\ldots=0$ may be evolved separately from a single expression whilst the index 1 remains fixed. But the question here relates to the possibility of evolving them four at a time in a certain definite order. And in effect if we examine the function $\mathrm{V}_{\mathrm{F}(\boldsymbol{\alpha} \boldsymbol{\beta})}$, we shall find that

$$
\mathrm{V}_{\mathrm{F}(\alpha \beta)}=\mathrm{P}_{h^{\prime}\left(\delta_{0}\right)_{n}}+\mathrm{P}_{g^{\prime}}+\mathrm{P}_{h\left(\delta_{. .}\right)_{n}}+\mathrm{P}_{g} ; .
$$

wherein I suppose $f_{\alpha}(\beta \gamma), f_{\beta}(\beta \gamma), \ldots$ to be denoted by $g_{\alpha}, g_{\gamma}, \ldots ; f_{\alpha}(\beta .),. f_{\beta}\left(\ddot{\beta}_{, .} \delta\right), \ldots$ by $h_{\alpha}, h_{\delta}, \ldots ;$ and the accent attached to the $g$ and $h$ to indicate, as before, a change of $a_{b}$ into $a_{i^{2}} *$. Whence it is clear that the four roots which com-

* It may be easily shown that

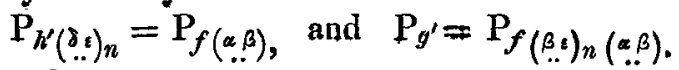

Observing that $h_{\alpha}=y_{\delta}+y_{\delta}+a_{\alpha} y_{\alpha}$, and that consequently $h_{\alpha}(\beta \delta)\left(\gamma_{. .}\right)=\mathfrak{c} h_{\omega}$ we immediately find from the theorem $\left(h_{\circ}\right)$

$$
\begin{aligned}
h_{\sigma}(\beta \delta)(\gamma \Xi) & =\left(\kappa h_{\tau}\right)(\delta \varepsilon)_{n} \\
& =h^{\prime}(\ldots \varepsilon)_{n} ;
\end{aligned}
$$

where $\sigma$ and $\tau$ may have more than one set of values assigned to them. Hence the same expression for $\mathbf{P}$ may be evolved from

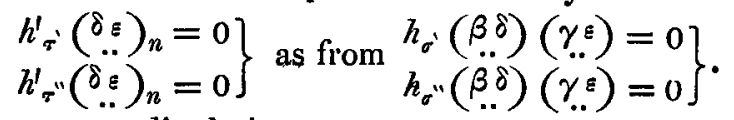

We must accordingly have

$$
\mathrm{P}_{h^{\prime}(\delta . .)_{n}}=\mathrm{P}_{h(\beta \delta .)(\gamma . .)}
$$


pose the function $V_{F(\alpha \beta)}$ do not all of them belong to one group, but must have come in pairs from two of the three groups (i.), (k.), (l.). Thus by the method of continuous substitutions we shall be conducted to an equation for $V_{F}$ of the form $\quad\left(V^{15}+C_{i} V^{14}+C_{2} V^{13}+\ldots+C_{15}\right)^{8}=0$, in which the coefficients $\mathrm{C}_{1}, \mathrm{C}_{2}, \ldots \mathrm{C}_{15}$ will be rational functions of $A_{1}, A_{9}, \ldots A_{5}$. And the roots of the equation $V^{15}$ $+\mathrm{C}_{1} \mathrm{~V}^{14}+\ldots=0$ will be expressible by

$$
\begin{aligned}
& =\mathrm{P}_{f(\gamma, .)} \\
& =\mathrm{P}_{f(\alpha \beta)} .
\end{aligned}
$$

See theorem (f.)

Again, observing that $g_{a}=y_{1}+y_{\gamma}+a_{a} y_{a}$, and therefore $g_{a}(\beta, \gamma)\left(\delta_{0}\right)=\mathfrak{c g}_{a} ;$ we find, as in the preceding case,

and thence

$$
\begin{aligned}
g_{\tau}(\beta \gamma)(\delta \varepsilon) & =\left(\mathfrak{r} g_{v}\right)(\gamma \varepsilon)_{n} \\
& =g_{g^{\prime}}{ }_{\nu}\left(\gamma_{.}\right)_{n}
\end{aligned}
$$

$$
\begin{aligned}
\mathbf{P}_{g^{\prime}(\gamma . s)_{n}} & =\mathrm{P}_{g(\beta \gamma)(\delta . .)} \\
& =\mathrm{P}_{f(\delta . .8)}
\end{aligned}
$$

Now if we apply the interchange $\left(\gamma_{. .}\right)$to the equations thus obtained, reflecting that, since the index $n$ may be suppressed,

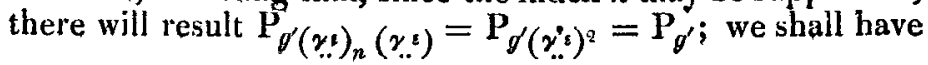

$$
\begin{aligned}
\mathrm{P}_{g^{\prime}} & =\mathrm{P}_{f(\delta . .)(\gamma . .)} \\
& =\mathrm{P}_{f(\gamma \delta)(\gamma \delta)} \\
& =\mathrm{P}_{f(\ldots \beta)(\gamma \delta)} ;
\end{aligned}
$$

the last of which functions will manifestly be equal to $P_{f\left(\beta_{t}\right)_{n}(\alpha \beta)}$; since $\left(\beta_{. .}\right)$and $\left(\gamma_{. .}^{\delta}\right)$ are complementary relatively to $f_{\alpha^{*}}$.

That $\mathrm{P}_{h^{\prime}(\delta 8)_{n}}$ and $\mathrm{P}_{g^{\prime}}$ do not belong to the same group, we may at once convince ourselves from considering that

$$
\mathbf{P}_{f}\left(\begin{array}{l}
\Lambda_{\alpha} \\
\Lambda_{1}
\end{array}\right)=\mathrm{P}_{i}, \quad \mathbf{P}_{g}\left(\begin{array}{l}
\Lambda_{\alpha} \\
\Lambda_{1}
\end{array}\right)=\cdot \mathbf{P}_{k}, \quad \mathbf{P}_{h}\left(\begin{array}{c}
\Lambda_{\alpha} \\
\Lambda_{1}
\end{array}\right)=\mathbf{P}_{l} ;
$$

where

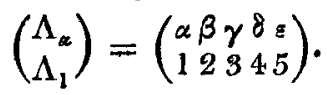

And in fact if we suppose $f$ to be changed into $i$,

and

$$
\mathrm{P}_{h^{\prime}(\Sigma . s)_{n}} \text { will become } \mathrm{P}_{l^{\prime}}\left(\frac{45}{.6)_{n}}\right.
$$

$$
\mathrm{P}_{g^{\prime}} \quad \cdots 2 \stackrel{\cdots}{\mathrm{P}_{2}} \mathrm{P}^{k^{\prime}}
$$




$$
\left.\begin{array}{l}
V_{\mathrm{I}}, \mathrm{V}_{\mathrm{I}(12)}, \ldots \mathrm{V}_{\mathrm{I}(15)}, \\
\mathrm{V}_{\mathrm{K}}, \mathrm{V}_{\mathrm{K}(1.2)}, \ldots \mathrm{V}_{\mathrm{K}(15)}, \\
\mathrm{V}_{\mathrm{L}}, \mathrm{V}_{\mathrm{L}(12)}, \ldots \mathrm{V}_{\mathrm{L}(15)} ;
\end{array}\right\} \cdot . \cdot \cdot
$$

which, except in particular cases, will be distinct one from another.

30. But if we designate by $W_{f^{\prime}(a b)(c d) . . .}$ the function

$$
\left(\mathrm{P}_{f}+\mathrm{P}_{f^{\prime}}\right)(\mathrm{a} . \mathrm{b})(\mathrm{c} \mathrm{d}) \ldots \text {, }
$$

which evidently does not admit of more than six different expressions, and observe that

$$
\mathrm{V}_{\mathrm{F}}=\mathrm{W}_{f^{\prime}}+\mathrm{W}_{f^{\prime}\left(\beta_{.}\right)_{n}}
$$

we shall see that the resolution of the equation $V^{15}+C_{1} V^{14}$ $+\ldots=0$ may be reduced to that of a determinate equation of six dimensions,

$$
W^{6}+D_{1} W^{5}+D_{2} W^{4}+\ldots+D_{6}=0
$$

the roots of which will be

$$
\left.\begin{array}{ll}
\mathbf{W}_{i}, & \mathbf{W}_{i(25)}, \\
\mathbf{W}_{k}, & \mathbf{W}_{k(35)_{n}} \\
\mathbf{W}_{l}, & \mathbf{W}_{l(45)_{n}}
\end{array}\right\} . \cdot . . . .
$$

31. Could we solve this equation for $W$, the roots of the general equation of the fifth degree might indeed be easily obtained. For from the expression for $\mathbf{P}_{f}+\mathbf{P}_{f^{\prime}}$ we might deduce that for $\mathrm{P}_{f}$ or $\mathrm{P}$. $p_{1}, p_{2}, p_{3}$ would thus become known. And by combining $x^{3}+p_{1} x^{2}+p_{2} x+p_{3}=y$ with $x^{5}+A_{1} x^{4}$ $+\mathrm{A}_{2} x^{3}+\ldots+\AA_{5}=0$, we should be conducted to

$$
x=q_{4}+q_{3} y+q_{2} y^{2}+q_{1} y^{3}+q_{0} y^{4} ;
$$

where $q_{4}, q_{3}, \ldots q_{0}$ are rational functions of $p_{1}, p_{2}, p_{3}$, or simply of $P$; and where

$$
y=\rho t+p^{4} u,
$$

$(\rho t)^{5}$ and $\left(\rho^{4} u\right)^{5}$ being, as is well known, the roots of the equation

$$
\left(t^{5}\right)^{2}+A_{5}^{\prime}\left(t^{5}\right)-\left(\frac{A_{2}^{\prime}}{5}\right)^{5}=0
$$

and consequently admitting of being expressed in terms of $A_{2}^{\prime}$ and $\mathrm{A}_{5}^{\prime}$, which also are rational functions of $p_{1}, p_{2}, p_{3}$.

\section{Section VI.}

32. We have not hitherto taken into consideration the forms of the functions denoted by $t$ and $u$. 
Now from the preceding number we perceive that

$$
\begin{aligned}
(\rho t)^{5} & =\mu+\alpha^{\prime} \sqrt{y} \\
\left(\rho^{4} u\right)^{5} & =\mu+\alpha^{\prime \prime} \sqrt{\nu}
\end{aligned}
$$

in which $\alpha^{\prime}$ and $\alpha^{\prime \prime}$ are the roots of the equation $\alpha^{2}-1=0$, and

Hence

$$
\mu=-\frac{A_{5}^{\prime}}{2}, \quad \nu=\left(\frac{A_{5}^{\prime}}{2}\right)^{2}+\left(\frac{A_{2}^{\prime}}{5}\right)^{5} .
$$

Again,

$$
(\rho t) \times\left(\rho^{4} u\right)=\rho^{5} \sqrt[5]{\mu^{2}-\nu}=-\frac{\mathbf{A}_{2}^{\prime}}{5} .
$$

$$
\begin{aligned}
y=\rho t+\rho^{4} u & =\rho t-\frac{A_{2}^{\prime}}{5 \rho t} \cdot \frac{(\rho t)^{4}}{(\rho t)^{4}} \\
& =(\rho t)-\frac{A_{2}^{\prime}}{5\left(\mu+\alpha^{\prime} \sqrt{v}\right)}(\rho t)^{4} ;
\end{aligned}
$$

and, as might be foreseen,

$$
\begin{aligned}
y=\rho^{4} u+\rho t & =\rho^{4} u-\frac{A_{2}^{\prime}}{5 \rho^{4} u} \cdot \frac{\left(\rho^{4} u\right)^{4}}{\left(\rho^{4} u\right)^{4}} \\
& =\left(\rho^{4} u\right)-\frac{A_{2}^{\prime}}{5\left(\mu+\alpha^{\prime \prime} \sqrt{\nu}\right)}\left(\rho^{4} u\right)^{4} .
\end{aligned}
$$

The equation (q.) may therefore, without altering the root $x$, be resolved into the two following equations,

$$
\left.\begin{array}{l}
x=r_{4}^{\prime}+r_{3}^{\prime}(\rho t)+r_{2}^{\prime}(\rho t)^{2}+r_{1}^{\prime}(\rho t)^{3}+r_{0}^{\prime}(\rho t)^{4}, \\
x=r_{4}^{\prime \prime}+r_{3}^{\prime \prime}\left(\rho^{4} u\right)+r_{2}^{\prime \prime}\left(\rho^{4} u\right)^{2}+r_{1}^{\prime \prime}\left(\rho^{4} u\right)^{3}+r_{0}^{\prime \prime}\left(\rho^{4} u\right)^{4} ;
\end{array}\right\}
$$

$r_{4}^{\prime}, r_{3}^{\prime}, \ldots r_{0}^{\prime}$ being rational with respect to $q_{4}, q_{3}, \ldots q_{0}, \mu$, $\sqrt{y}, \mathrm{~A}_{2}^{\prime}$; and $r^{\prime}$ becoming $r^{\prime \prime}$ when $\alpha^{\prime}$ is changed into $x^{\prime \prime}$.

33. If now in the equation

$$
x=r_{4}+r_{3} v+r_{2} v^{2}+r_{1} v^{3}+r_{0} v^{4}
$$

which may represent either of the equations (r.), we write ${ }^{\zeta} v$ instead of $v$; taking $\zeta$ successively equal to $0,1,2,3,4$, we shall, if

$$
r_{n} v^{4-n}=\Theta_{4-n}
$$

arrive at the system of equations

$$
\begin{aligned}
& x_{\alpha}=\theta_{0}+\theta_{1}+\theta_{2}+\theta_{3}+\theta_{4}, \\
& x_{\beta}=\theta_{0}+\imath \theta_{1}+\imath^{2} \theta_{2}+\imath^{3} \theta_{3}+\imath^{4} \theta_{4}, \\
& x_{\gamma}=\theta_{0}+\iota^{2} \theta_{1}+\imath^{4} \theta_{2}+\iota \theta_{3}+\imath^{3} \theta_{4} \text {, } \\
& x_{\delta}=\theta_{0}+\imath^{3} \theta_{1}+\imath \theta_{2}+\iota^{4} \theta_{3}+\imath^{2} \theta_{4} \text {, } \\
& x_{s}=\theta_{0}+\iota^{4} \theta_{1}+\imath^{3} \theta_{2}+\imath^{2} \theta_{3}+\imath \theta_{4} ;
\end{aligned}
$$

from which there will result 


$$
\begin{aligned}
& \iota^{5 n} x_{\alpha}=\ldots+{ }^{(5+0) n} \theta_{n}+\ldots, \\
& \iota^{4 n} x_{\beta}=\ldots+{ }^{(4+1) n} \theta_{n}+\ldots, \\
& \iota^{3 n} x_{\gamma}=\ldots+{ }^{(3+2) n} \theta_{n}+\ldots, \\
& \iota^{2 n} x_{\delta}=\ldots+{ }^{(2+3) n} \theta_{n}+\ldots, \\
& \iota^{n} x_{\iota}=\ldots+{ }^{(1+4) n} \theta_{n}+\ldots ;
\end{aligned}
$$

the successive coefficients of $\Theta_{n+k}$ being $\imath^{5 n}, 1^{5 n+h}, 1^{5 n+2 h}$, $b^{5 n+3 h}, b^{5 n+4 h}$, or $\left(b^{h}\right)^{0},\left(b^{h}\right)^{1},\left(\imath^{h}\right)^{2},\left(\iota^{h}\right)^{3},\left(b^{h}\right)^{4}$.

And if we reflect that the system $(\theta$.) will remain unaltered

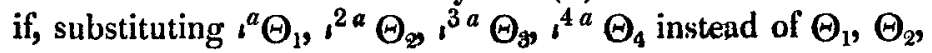
$\Theta_{3}, \Theta_{4}$ respectively, we make suitable substitutions among $x_{\alpha}$, $x_{\beta}, x_{\gamma}, x_{\gamma}, x_{1}$; and that $\Theta_{n}$, or $v_{4-n} v^{n}$, inust be such as to admit of being equated either to $r_{4-n}^{\prime}(\rho t)^{n}$ or to $r_{4-n}^{\prime \prime}\left(\rho^{4} u\right)^{n}$; we shall have little difficulty in perceiving that

$$
\begin{aligned}
& \left\{\Theta_{n}^{5}-\frac{1}{5^{5}}\left(x_{\alpha}+i^{4 n} x_{\beta}+\imath^{3 n} x_{\gamma}+\imath^{2 n} x_{\delta}+\imath^{n} x_{\xi}\right)^{5}\right\} \times \\
& \left\{\Theta_{n}^{5}-\frac{1}{5^{5}}\left(x_{\alpha}+\imath^{n} x_{\beta}+\iota^{2 n} x_{\gamma}+\imath^{3 n} x_{\delta}+\iota^{4 n} x_{\S}\right)^{5}\right\}=0 .
\end{aligned}
$$

34. We are thus permitted to assume

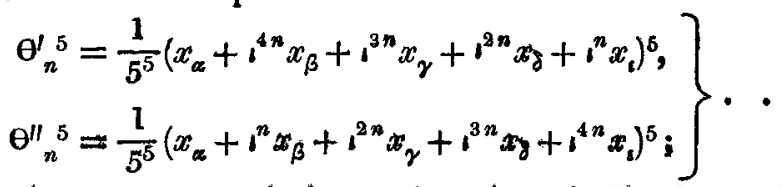

$\theta_{n}^{\prime}{ }_{n}^{5}, \theta^{\prime \prime}{ }_{n}^{5}$ being respectively equal to $r_{4-n}^{s}(\rho t)^{n}, r^{n}{ }_{4-n}\left(\rho^{4} u\right)^{n}$.

35. It follows therefore that

Whence we deduce

$$
\theta_{n}^{\prime 5}=\theta_{4 n}^{\prime \prime} \text {. . . . . . . }
$$

$$
\begin{array}{ll}
\Theta_{1}^{\prime}=\Theta^{\prime \prime}{ }_{4}^{5}, & \Theta_{2}^{\prime 5}=\Theta^{\prime \prime}{ }_{3}^{5}, \\
\Theta_{4}^{\prime 5}=\Theta^{\prime \prime}{ }_{1}^{5}, & \Theta_{3}^{\prime 5}=\Theta_{2}^{\prime \prime} ;
\end{array}
$$

observing that $\Theta^{5}{ }_{5 a+b}=\Theta_{b}{ }^{5}$.

\section{Secrion VII.}

36. Let us now consider $\Theta_{n}$ in relation to the different values of $P$. To indicate that the expression which may take the place of $\Theta_{n}$ is a function of $P_{f(a b)(c d)}$, I annex to $\Theta_{n}$ the index of $P$. 'Thus

$$
\Theta_{n, f(a b)(c d)}
$$


will denote a function of that value of $P$, the index of which is $f(a b)(c d)$... have

37. It is evident from the equations (s.) and (to) that we may

$$
\left.\begin{array}{c}
\Theta_{n, f(\mathrm{ab})(\mathrm{cd}) . . .}=\frac{\iota^{5}}{5}\left(x_{\alpha}+\iota^{4 n} x_{\beta}+\iota^{3 n} x_{\gamma}+\iota^{2 n} x_{\delta}+i^{n} x_{\varepsilon}\right)(\mathrm{ab})(\mathrm{cd}) . . \\
\text { if } \quad \Theta_{n, f(. . . \mathrm{ab})(\mathrm{cd}) . .}=\iota^{5}\left(\Theta_{n, f}^{\prime}\right)(\mathrm{ab})(\mathrm{cd}) . .
\end{array}\right\}
$$

or

$\left.\begin{array}{c}\Theta_{n, f(\mathrm{ab})(\mathrm{cd}) . . .}=\frac{\iota^{n}}{5}\left(x_{a}+\iota^{n} x_{\beta}+\iota^{2 n} x_{\gamma}+\iota^{3 n} x_{\delta}+\iota^{4 n} x_{\mathrm{s}}\right)\left(\begin{array}{l}\mathrm{ab} \\ . .\end{array}\right)(\mathrm{cd}) . . . \\ \text { if } \quad \Theta_{n, f(a \mathrm{ab})(\mathrm{cd}) . .}=\iota^{n}\left(\Theta^{\prime \prime}{ }_{n, f}\right)(\mathrm{ab})(\mathrm{cd}) . .\end{array}\right\}$

wherein $\zeta$ and $\eta$ are not independent of (ab) (cd)... This theorem is remarkable not only for its hypothetical character, but also for being composed of two branches.

38. If we suppose the operation denoted by (a b) (cd) ... to take the particular form ( $a b)(a b)$, there will result from the first branch,

$$
\left.\begin{array}{c}
\Theta_{n, f}=\frac{\iota_{1}}{5}\left(x_{\alpha}+\iota^{4 n} x_{\beta}+\iota^{3 n} x_{\gamma}+\imath^{2 n} x_{\delta}+\imath^{n} x_{\S}\right) \\
\text { if } \quad \Theta_{n, f}=\iota_{1} \Theta_{n, f}^{\prime}
\end{array}\right\} .
$$

Further, if, observing that $\mathrm{P}_{f(\beta, .)(\gamma \delta)}=\mathrm{P}_{f}$, and consequently $\Theta_{n, f\left(\beta_{3}\right)(\gamma \delta)}=\Theta_{n, f}$, we suppose (a b) (c... .. to become $(\beta \varepsilon)\left(\gamma_{.}^{\delta}\right)$, we shall have

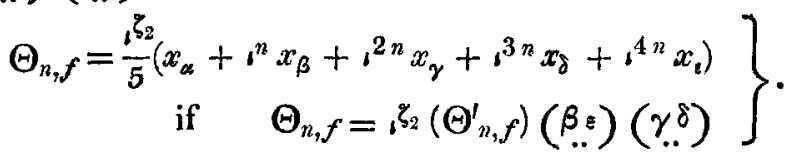

Lastly, since $\mathrm{P}_{f(\alpha \beta)(\gamma \varepsilon)}=\mathrm{P}_{f}$, the same branch will give

$$
\left.\begin{array}{c}
\Theta_{n, f}=\frac{\zeta_{3}+4 n}{5}\left(x_{\alpha}+\iota^{n} x_{\beta}+\iota^{2 n} x_{\gamma}+\iota^{3 n} x_{\delta}+\iota^{4 n} x_{\varepsilon}\right) \\
\text { if } \quad \Theta_{n, f}=\iota^{\zeta_{3}}\left(\Theta_{n, f}^{\prime}\right)(\alpha \beta)\left(\gamma_{.}\right)
\end{array}\right\} . \quad\left(v_{3}\right)
$$

And analogous results will be obtainable from the other branch (w.).

Now we see that $\left(\mathrm{v}_{1}\right)$ cannot generally apply to $\Theta^{\prime \prime}{ }_{n, f}$ (since the condition $\Theta_{n, f}^{\prime \prime}=r^{\zeta} \Theta_{n, f}^{\prime}$ cannot be satisfied without inducing certain relations among $\left.x_{1}, x_{2}, \ldots x_{5}^{*}\right)$; but that it will

* See the equation (u.). 
apply to $\Theta_{n, f}^{\prime}$. On the contrary, $\left(v_{2}\right)$ and $\left(v_{3^{*}}\right)$ will be clearly applicable to $\Theta^{\prime \prime}{ }_{n, f}$, and not to $\Theta_{n, f}^{\prime}$.

If we take $\zeta_{1}=\zeta_{2}=0$, we shall have

$$
\left.\begin{array}{rl}
\Theta_{n, f}^{\prime} & =\frac{1}{5}\left(x_{\alpha}+\iota^{4 n} x_{\beta}+\iota^{3 n} x_{\gamma}+\iota^{2 n} x_{\delta}+\iota^{n} x_{\iota}\right), \\
\Theta^{\prime \prime} & =\frac{1}{5}\left(x_{\alpha}+\iota^{n} x_{\beta}+\iota^{2 n} x_{\gamma}+\iota^{3 n} x_{\delta}+\iota^{4 n} x_{\imath}\right),
\end{array}\right\} .
$$

But having decided upon thus fixing the meanings of $\Theta_{n, f}^{\prime}$ and $\Theta_{n, f}^{\prime \prime}$, we must be careful, in evolving particular forms

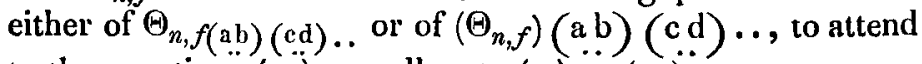
to the equations $(\mathrm{x}$.$) as well as to (\mathrm{v}$.$) or (w$.$) .$

There may also subsist

and

$$
\left.\begin{array}{rl}
\Theta^{\prime}{ }_{n, f\left(\beta_{\varepsilon}\right)} & =\left(\Theta^{\prime \prime}{ }_{n, f}\right)\left(\gamma_{. .}^{\delta}\right), \\
\Theta^{\prime \prime}{ }_{n, f\left(\beta_{.}\right)} & =\left(\Theta^{\prime \prime}{ }_{n, f}\right)\left(\beta_{. .}\right),
\end{array}\right\} . . . . \cdot .
$$

$$
\left.\begin{array}{rl}
\Theta^{\prime} & \left.{ }_{n, f(\alpha \beta)}=\left(\Theta^{\prime \prime}{ }_{n, f}\right)\left(\gamma_{. .}\right)^{\prime}\right), \\
\Theta^{\prime \prime}{ }_{n, f(\alpha \beta)} & =\left(\Theta^{\prime \prime}{ }_{n, f}\right)(\alpha \beta),
\end{array}\right\} .
$$

$\Theta_{n, f\left(\beta_{. .}\right)}$being equal to $\Theta_{n, f(\gamma . .)}$, and $\Theta_{n, f(\alpha \beta)}$ to $\Theta_{n, f\left(\gamma^{s}\right)}$.

\section{Section VIII.}

39. Again, if we examine the equation

$$
\Theta_{n}\left(\beta_{. .}\right)=\frac{1}{5}\left(x_{\alpha}+\iota^{n} x_{\beta}+\iota^{3 n} x_{\gamma}+\iota^{2 n} x_{\delta}+\iota^{4 n} x_{s}\right),
$$

on designating, for the moment, $\Theta_{n}\left(\beta_{. .}\right)$by $\mathbf{I}_{n}$, we shall perceive that

$$
\begin{aligned}
& I_{0}+I_{1}+I_{2}+I_{3}+I_{4}=x_{\alpha}=\Theta_{0}+\Theta_{1}+\Theta_{2}+\Theta_{3}+\Theta_{4}, \\
& I_{0}+\imath^{4} I_{1}+\imath^{3} I_{2}+\imath^{2} I_{3}+\imath I_{4}=x_{\beta}=\Theta_{0}+\imath \Theta_{1}+\imath^{2} \Theta_{2}+\imath^{3} \Theta_{3}+\imath^{4} \Theta_{4} \text {, } \\
& \mathrm{I}_{0}+\imath^{2} \mathrm{I}_{1}+\imath^{4} \mathrm{I}_{2}+\imath \mathrm{I}_{3}+\imath^{3} \mathrm{I}_{4}=x_{\gamma}=\Theta_{0}+\imath^{2} \Theta_{1}+\imath^{4} \Theta_{2}+\imath \Theta_{3}+\imath^{3} \Theta_{4} \text {, } \\
& \mathrm{I}_{0}+\imath^{3} \mathrm{I}_{1}+\imath \mathrm{I}_{2}+\imath^{4} \mathrm{I}_{3}+\imath^{2} \mathrm{I}_{4}=x_{\delta}=\Theta_{0}+\imath^{3} \Theta_{1}+\imath \Theta_{2}+\imath^{4} \Theta_{3}+\imath^{2} \Theta_{4} \text {, } \\
& I_{0}+\imath_{1}+i^{2} I_{2}+i^{3} I_{3}+i^{4} I_{4}=x_{\imath}=\Theta_{0}+i^{4} \Theta_{1}+i^{3} \Theta_{2}+\imath^{2} \Theta_{3}+\imath^{2} \Theta_{4} ;
\end{aligned}
$$

$$
\begin{gathered}
5 \Theta_{n}=\left(3+\iota^{2 n}+\iota^{3 n}\right) I_{n}+\left\{1+2\left(\iota^{2 n}+\iota^{3 n}\right)\right\} I_{2 n} \\
+\left\{1+2\left(\iota^{n}+\iota^{4 n}\right)\right\} I_{3 n}+\left(3+\iota^{n}+\iota^{4 n}\right) I_{4 n} ;
\end{gathered}
$$

which connects one system with the other.

Hence if we denote

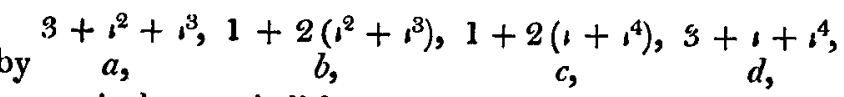

respectively, we shall have 


$$
\begin{aligned}
& \Theta_{1}=\frac{1}{5}\left(a \mathrm{I}_{1}+b \mathrm{I}_{2}+c \mathrm{I}_{3}+d \mathrm{I}_{4}\right), \\
& \Theta_{2}=\frac{1}{5}\left(d \mathrm{I}_{2}+c \mathrm{I}_{4}+b \mathrm{I}_{1}+a \mathrm{I}_{3}\right), \\
& \Theta_{3}=\frac{1}{5}\left(d \mathrm{I}_{3}+c \mathrm{I}_{1}+b \mathrm{I}_{4}+a \mathrm{I}_{2}\right), \\
& \Theta_{4}=\frac{1}{5}\left(a \mathrm{I}_{4}+b \mathrm{I}_{3}+c \mathrm{I}_{2}+d \mathrm{I}_{1}\right) .
\end{aligned}
$$

Elevating each of these eight functions to the fifth power, I now express, as before, $\Theta_{n}$ as a function of $\mathrm{P}_{f}$; and $\mathrm{I}_{n}$ as a function of $\mathrm{P}_{f\left(\beta_{s}\right)}$; and observing that $\Theta_{n, f}^{\prime}=\Theta^{\prime \prime}{ }_{4 n, f}$, and that $\left.\left(\Theta_{n, f}^{\prime}\right)\left(\beta_{. .}\right)=\Theta^{\prime}{ }_{n, f\left(\beta_{\varepsilon}\right)}{ }^{*},\left(\Theta^{\prime \prime}{ }_{n, f}\right)\left(\beta_{. .}\right)=\Theta^{\prime \prime}{ }_{n, f(\beta \varepsilon}\right), \mathrm{I}$ find $\Theta_{1, f}^{15}+\ddot{\Theta}_{2, f}^{\prime 5}+\Theta_{2, f}^{\prime \prime 5}+\Theta_{1, f}^{\prime \prime 5}=$

$$
\begin{aligned}
& \frac{1}{5^{5}}\left(a \Theta_{1, f\left(\beta_{.}\right)}^{\prime}+b \Theta_{2, f\left(\beta_{\xi}\right)}^{\prime}+c \Theta_{2, f\left(\beta_{\xi}\right)}^{\prime \prime}+d \Theta^{\prime \prime}{ }_{1, f\left(\beta_{,}\right)}\right)^{5} \\
& +\frac{1}{5^{5}}\left(d \Theta_{2, f\left(\beta_{.8}\right)}^{\prime}+c \Theta_{1, f\left(\beta_{.8}\right)}^{\prime \prime}+b \Theta_{1, f\left(\beta_{. .}\right)}^{\prime}+a \Theta_{2, f\left(\beta_{. .}\right)^{\prime}}\right)^{5} \\
& +\frac{1}{5^{5}}\left(d \Theta^{\prime \prime}{ }_{2, f\left(\beta_{.}\right)}+c \Theta_{1, f\left(\beta_{\xi}\right)}^{\prime}+b \Theta^{\prime \prime}{ }_{1, f\left(\beta_{.}\right)}+a \Theta_{2, f\left(\beta_{\xi}\right)}^{\prime}\right)^{5} \\
& +\frac{1}{5^{5}}\left(a \Theta_{1, f\left(\beta_{.}\right)}^{\prime \prime}+b \Theta_{2, f\left(\beta_{\varepsilon}\right)}^{\prime \prime}+c \Theta_{2, f\left(\beta_{\varepsilon}\right)}^{\prime}+d \Theta_{1, f\left(\beta_{.8}\right)}^{\prime}\right)^{5} ;
\end{aligned}
$$

of which the first member is a rational function of $\mathrm{P}_{f} \dagger$; and the second is a function, but not a rational one, of $P_{f(\beta, .)}$ another root of the equation for $P$.

In this theorem we may evidently change $f$ successively into $i, k, l ; f\left(\beta_{. .}\right)$successively becoming $i\left(\begin{array}{c}25 \\ . .\end{array}\right), k\left(\begin{array}{c}35 \\ . .\end{array}\right), l(4.5)$. We may also write $f\left(\beta_{. \varepsilon}\right)$ and $f^{\prime}$ instend of $f$. But for certain values of the letters in (ab) (c. d) ... the equation (aa.) will be discontinuous in consequence of the hypothetical character of the fundamental theorem $(v, w$.$) .$

40. Writing in the expression for $\Theta_{1, f}^{\prime 5}+\Theta^{15}{ }_{2, f}+\Theta^{\prime 15}{ }_{2, f}$ $+\Theta^{\prime \prime 5}{ }_{1, f}, b^{\zeta n} \Theta^{\prime}{ }_{n, f(\beta, .)}$ instead of $\Theta^{\prime}{ }_{n, f(\beta, .)}$, and $\left.\iota^{4 n} \Theta^{\prime \prime}{ }_{n, f(\beta,}\right)$ instead of $\Theta^{\prime \prime}{ }_{n, f\left(\beta_{.}\right)}$; and denoting the function which will

* Compare the first of the equations (y.) with the equations (x.) and $\left(v_{2}.\right)$.

+ For $\theta_{1, f}^{\prime 5}+\ldots$ is evidently included in the form

or

$$
M+\sqrt{N}+M^{\prime}+\sqrt{ } \bar{N}^{\prime}+M^{\prime}-\sqrt{ } \bar{N}^{\prime}+M-\sqrt{N},
$$

where $M$ and $M^{\prime}$ are rational functions of $P_{f}$. 
thence arise by $\zeta \Xi$, we shall, on taking $\zeta$ successively equal to $0,1,2,3,4$, obtain the five functions ${ }_{0} \Xi,{ }_{1} \Xi,{ }_{2} \Xi,{ }_{3} \Xi,{ }_{4} \Xi$; the first of which, ${ }_{0} \Xi$, will be equal to $\Theta^{15}{ }_{1, f}+\Theta_{2, f}^{15}+\Theta_{2, f}$ $+\Theta^{\prime \prime 5} 1, f$.

We may therefore form an equation of five dimensions,

$$
\left(\Xi-{ }_{0} \Xi\right)\left(\Xi-{ }_{1} \Xi\right) .(\Xi-\Xi)=0 ; . . \quad \text {. (ab.) }
$$

the coefficients of which, when arranged according to the powers of $\Xi$, shall be rational functions of $P_{f\left(\beta_{\xi}\right)^{\cdot}}$. For, in consequence of the symmetric manner in which $a^{i}$ and $\alpha^{\prime \prime}$ will enter into these coefficients, the symbol $\sqrt[2]{ }$, as well as $\sqrt[5]{ }$, will disappear from the calculus*.

41. Now $\Theta_{1, f}^{\prime 5}+\ldots+\Theta_{1, f}^{15}{ }_{1,}$, as we have already stated, a rational function of $\mathbf{P}_{f}$, and cannot therefore, when the roots $x_{1}, x_{2}, \ldots x_{5}$ change places among themselves, receive more than twelve different values. If, indeed, we consider that $\left(\Theta^{15}{ }_{1, f}+\ldots+\Theta^{15}{ }_{1, f}\right)\left(\beta_{. \gamma}\right)\left(\delta_{. .} \varepsilon\right)=\left(\Theta^{15}{ }_{1, f}+\ldots+\Theta^{\prime 15}{ }_{1, f}\right)\left(\gamma_{.}^{\delta}\right)$, while $\mathrm{P}_{f(\beta \gamma)(\delta .)}$ is not equal to $\mathrm{P}_{f(\gamma \delta)}$, but to $\mathrm{P}_{f^{\prime}\left(\gamma^{\delta}\right)}$; we shall instantly perceive that $\Theta^{\prime 5} 1, f+\ldots+\Theta^{\prime \prime 5} 1, f$ must admit of becoming a root of a determinate equation of the $\frac{12}{2}$ th degree expressible by

* We see at once that we are permitted to suppose ${ }_{0} \Xi=\psi_{1}\left(\alpha^{\prime}, \alpha^{\prime \prime}\right)+\psi_{2}\left(\alpha^{\prime}, \alpha^{\prime \prime}\right)+\psi_{2}\left(\alpha^{\prime \prime}, \alpha^{\prime}\right)+\psi_{1}\left(\alpha^{\prime \prime}, \alpha^{\prime}\right)$, ${ }_{0} \Xi$ will therefore belong to the class of expressions

There may also subsist

$$
{ }_{0} \Psi\left(\alpha^{\prime}, \alpha^{\prime \prime}\right)+{ }_{0} \Psi\left(\alpha^{\prime \prime}, \alpha^{\prime}\right) \text {. }
$$

$$
\begin{aligned}
& \left.1={ }^{1} \psi_{1}\left(\alpha^{\prime}, \alpha^{\prime \prime}\right)+{ }^{1} \psi_{2}\left(\alpha^{\prime}, \alpha^{\prime \prime}\right)+{ }^{4} \psi_{2}\left(\alpha^{\prime \prime}, \alpha^{\prime}\right)+{ }^{4} \psi_{1}\left(\alpha^{\prime \prime}, \alpha^{\prime}\right),\right\} \\
& \left.{ }_{4} \exists={ }^{4} \psi_{1}\left(\alpha^{\prime}, \alpha^{\prime \prime}\right)+{ }^{4} \psi_{2}\left(\alpha^{\prime}, \alpha^{\prime \prime}\right)+{ }^{1} \psi_{2}\left(\alpha^{\prime \prime}, \alpha^{\prime}\right)+{ }^{1} \psi_{1}\left(\alpha^{\prime \prime}, \alpha^{\prime}\right) .\right\} \\
& \text { and } \\
& \left.\begin{array}{l}
{ }_{9} \equiv{ }^{2} \psi_{1}\left(\alpha^{\prime}, \alpha^{\prime \prime}\right)+{ }^{2} \psi_{2}\left(\alpha^{\prime}, \alpha^{\prime \prime}\right)+{ }^{3} \psi_{2}\left(\alpha^{\prime \prime}, \alpha^{\prime}\right)+{ }^{3} \psi_{1}\left(\alpha^{\prime \prime}, \alpha^{\prime}\right), \\
{ }_{3} \Xi={ }^{3} \psi_{1}\left(\alpha^{\prime}, \alpha^{\prime \prime}\right)+{ }^{3} \psi_{2}\left(\alpha^{\prime}, \alpha^{\prime \prime}\right)+{ }^{2} \psi_{2}\left(\alpha^{\prime \prime}, \alpha^{\prime}\right)+{ }^{2} \psi_{1}\left(\alpha^{\prime \prime}, \alpha^{\prime}\right) ;
\end{array}\right\}
\end{aligned}
$$

as is ovident. If, therefore, we suppose ${ }_{1} \Xi$ and ${ }_{2} \Xi$ to be denoted by

respectively, ${ }_{4}$ and ${ }_{3}$ must take the forms

$$
{ }_{1} \Psi\left(\alpha^{\prime}, \alpha^{\prime \prime}\right),{ }_{2} \Psi\left(\alpha^{\prime}, \alpha^{\prime \prime}\right)
$$

$$
{ }_{1} \Psi\left(\alpha^{\prime \prime}, \alpha^{\prime}\right), \quad{ }_{2} \Psi\left(\alpha^{\prime \prime}, \alpha^{\prime}\right) \text {. }
$$

Whence it appears that $\alpha^{\prime}$ and $\alpha^{\prime \prime}$ are involved symmetrically in the equation (a b.).

There are some other results connected with the equation (a b.) which I should like to verify, but I have no time to do so now. 
$\left.\begin{array}{c}\left(\Xi-\Xi_{f}\right)\left(\Xi-\Xi_{g}\right)\left(\Xi-\Xi_{h}\right) \\ \times\left(\Xi-\Xi_{f\left(\beta_{s s}\right)}\right)\left(\Xi-\Xi_{g\left(y_{. s}\right)}\right)\left(\Xi-\Xi_{h\left(\delta_{s}\right)}\right)=0^{*} ;\end{array}\right\}$ (a c.) $\Xi_{f}, \Xi_{g}, \ldots \Xi_{h\left(\delta_{i}\right)}$ representing rational functions of $\mathrm{P}_{f}, \mathrm{P}_{g}, \ldots$ $\mathbf{P}_{h(\delta)}$

4.2. Comparing the equations (a b.) and (a c.), we are now conducted to an equation of the form

$$
\Xi_{f}-\mathfrak{t}\left(P_{f(\beta, .)}\right)=0 \text {; }
$$

in which $r$ is expressive of a rational function; that is, we find

$$
\begin{gathered}
a_{11}+a_{10} P_{f}+a_{9} P_{f}^{2}+\ldots+a_{0} P_{f}^{11}=b_{11}+b_{10} P_{f(\beta . .)} \\
\left.+b_{9} P_{f(\beta .)}^{2}+\ldots+b_{0} P_{f(\beta, .)}^{11}\right)
\end{gathered}
$$

$a_{11}, a_{10}, \ldots a_{0}, b_{11}, b_{10}, \ldots b_{0}$ being symmetric functions of $x_{1}$, $x_{2}, \ldots x_{5}$.

And comparing this equation with

there will result

$$
\mathrm{P}_{f}^{12}+\mathrm{B}_{1} \mathrm{P}_{f}^{11}+\mathrm{B}_{2} \mathbf{P}_{f}^{10}+\ldots+\mathrm{B}_{12}=0
$$

$$
\mathbf{P}_{f}={ }^{\mathfrak{l}} \mathfrak{l}\left(\mathbf{P}_{f(\beta, .)}\right) ;
$$

where ${ }^{1} r$ will represent a rational function.

We must also have, since in the theorem (a a.) we are permitted to write $f(\beta \varepsilon)$ instead of $f$,

and therefore

$$
\begin{aligned}
& \Xi_{f\left(\beta_{.:}\right)}-\mathfrak{r}\left(\mathrm{P}_{f}\right)=0, \\
& \mathrm{P}_{f\left(\beta_{.}\right)}={ }^{\mathrm{l}} \mathfrak{r}\left(\mathrm{P}_{f}\right) ;
\end{aligned}
$$

$$
\mathbf{P}_{f}={ }^{\mathbf{l}} \mathfrak{r}\left(\mathbf{l}_{\mathfrak{r}}\left(\mathbf{P}_{f}\right)\right) \text {. }
$$

43. Similarly, on considering that $\mathbf{P}_{f^{\prime}}$ may be expressed as a rational function of $P_{f^{\prime}}$ and $P_{f^{\prime}\left(\beta_{.1}\right)}$ of $P_{f\left(\beta_{. .}\right)}$(41.), we shall see that

and thence

$$
\begin{aligned}
& \mathbf{P}_{f}={ }_{1} \mathbf{R}\left(\mathbf{P}_{f}+\mathbf{P}_{f^{\prime}}\right)={ }_{1} \mathbf{R}\left(\mathbf{W}_{f^{\prime}}\right) \text {, } \\
& \mathrm{P}_{f}={ }_{2} \mathrm{R}\left(\mathrm{P}_{f(\beta \varepsilon)}+\mathrm{P}_{f^{\prime}\left(\beta_{\ell} g\right)}\right)={ }_{2} \mathrm{R}\left(\mathrm{W}_{f^{\prime}\left(\beta_{\ell} g\right.}\right) \text {; }
\end{aligned}
$$

$$
{ }_{1} R\left(W_{f^{\prime}}\right)={ }_{2} R\left(W_{f^{\prime}(\beta . . s)}\right) ;
$$

${ }_{1} R$ and ${ }_{2} R$ representing rational functions.

- The equation (a c.) must, in fact, be capable of coinciding with the celebrated equation of the sixth degree, by which Vandermonde and Lagrange were stopped in their researches on the solution of algebraic equattions of the fifth degree. 
And combining this equation with

$$
\mathrm{W}_{f^{\prime}}{ }^{6}+\mathrm{D}_{1} \mathrm{~W}_{f^{5}}+\ldots+\mathrm{D}_{6}=0
$$

we shall ultimately obtain

$$
\mathbf{W}_{f^{\prime}}={ }^{1} \mathrm{R}\left(\mathrm{W}_{f^{\prime}\left(\beta_{g}\right)}\right)^{*}
$$

${ }^{1} \mathrm{R}$ also being expressive of a rational function.

The equation for $W$ will therefore belong to a class of equations of the sixth degree, the resolution of which can, as Abel $\dagger$ has shown, be effected by means of equations of the second and third degrees.

Whence I infer the possibility of solving by a finite combination of radicals and rational functions the general equation of the fifth degree. (31.)

44. The equation of which

$$
\mathbf{W}_{f^{n}}+{ }^{1} \mathbf{R}\left(\mathbf{W}_{f^{\prime \prime}}\right)
$$

is a root will evidently be of the third degree. For omitting the parentheses connected with ${ }^{1} R$, we see that

$$
{ }^{1} \mathbf{R} \mathbf{W}_{f^{\prime}}={ }^{1} \mathbf{R}^{2} \mathbf{W}_{f^{\prime \prime}(\beta, .)}=\mathbf{W}_{f^{\prime}(\beta ! .)} \text {, }
$$

the exponent, as is usual, indicating a repetition of an operation; and that consequently the root in question will not be affected by writing $f^{\prime}\left(\beta_{\varepsilon}\right)$ instead of $f^{\prime}$.

We must also have

$$
\left(\mathrm{W}_{f^{\prime}}+{ }^{1} \mathrm{R} \mathrm{W}_{f^{\prime}}\right)(\mathrm{a} \cdot \mathrm{b})(\mathrm{c} \cdot \mathrm{d}) \ldots=\left(\mathrm{V}_{\mathrm{F}}\right)(\mathrm{a} . . .)(\mathrm{cd}) \ldots,
$$

when (ab) (cd)... takes the form (ab) (ab); but not for all values of $a, b, c, d, \ldots$ since the method of continuous substitutions will not generally be applicable to processes based upon the theorem ( $v, w$. ), which is, as we must remember, hypothetical in itself.

Hence I conclude that there will be an equation of the third degree with given coefficients simultaneous with the equation $V^{15}+C_{1} V^{14}+\ldots=0$, which cannot be depressed below the 15th degree without inducing certain relations among $A_{1}, A_{2}$, .. $A_{5} \neq$.

* We shall have, as before,

$$
\mathrm{w}_{f^{\prime}}={ }^{\mathrm{l}} \mathrm{R}\left(\mathrm{l} R\left(\mathrm{w}_{f^{\prime}}\right)\right) \text {. }
$$

† In a memoir "Sur une classe particulière d'Equations résolubles algébriquement." Crelle's Journal, vol. iv. p. 131.

$\ddagger$ All this will be more readily understood from considering that, in expanding any function whatever of $x+h$ two independent quantities, we do not necessarily obtain an expression symmetric with respect to $x$ and $h$, and such as to admit of an interchange between them. In fact, instead of the 
45. Again, if we consider that $P_{f^{\prime}(\beta s)}, P_{f^{\prime}}$ are rational functions of $P_{f(B, .)}, P_{f}$, using $R$ as the characteristic, we shall have

and

$$
\mathrm{P}_{f^{\prime}(\beta \xi)}=\mathrm{R} \mathrm{P}_{f(\beta, .)}=\mathrm{R}^{1} \mathfrak{r} \mathrm{P}_{f}
$$

$$
P_{f^{\prime}(\beta, .)}={ }^{1} \mathfrak{r} P_{f^{\prime}}={ }^{1} \mathfrak{r} R P_{f^{*}}
$$

Hence the equation for $P$ will be such that

$$
\mathbf{R}^{1} \mathfrak{r} \mathbf{P}_{f}={ }^{1} \mathfrak{r} R P_{f}
$$

and if we further consider that there must subsist an equation analogous to (aa.) when each of the remaining roots is combined with $\mathrm{P}_{f}$, we shall find ourselves conducted to another class of equations solved by $\mathrm{Abel}$ in the memoir just alluded to.

46. Lastly, $\Xi_{f(\beta, .)}$ being (4i1.) a rational function of $\mathrm{P}_{f(\beta, .)}$, we obtain (42.)

$$
\begin{aligned}
\Xi_{f} & =\mathfrak{r}^{\prime} \Xi_{f(\beta, s)}, \\
\Xi_{f(\beta, .)} & =\mathfrak{r}^{\prime} \Xi_{f} ;
\end{aligned}
$$

$r$ ' indicating a rational function.

The equation for the celebrated function $\Xi$ will therefore belong to the same class as that for $W$, and nust consequently adnit of a similar solution. Meyer Hirsch, in endeavouring to solve this equation, alighted upen the equation of the 15th degree analogous to $\mathrm{V}^{15}+\mathrm{C}_{1} \mathrm{~V}^{14}+\ldots=0$.

At some future time I hope to return to this subject, and to discuss the resolution of the trinomial equation

$$
x^{5}+\mathrm{A}_{4} x+\mathrm{A}_{5}=0 \text {; }
$$

to which very simple form the general equation of the fifth

absolute or unconditional theorem of Taylor, we ought to have, as the discussion in the text first led me to perceive,

$$
\left.\begin{array}{l}
f(x+h)=f(x)+f^{\prime}(x) \frac{h}{1}+f^{\prime 2}(x) \frac{h^{2}}{1.2}+f^{3}(x) \frac{h^{3}}{1.2 .3}+. . \\
\text { if } \quad\left(f^{i+1}(x)+f^{i+2}(x) \frac{h}{1.2}+f^{\prime+3}(x) \frac{h^{2}}{1.2 .3}+. .\right) \times h\left(\begin{array}{l}
h \\
0
\end{array}\right)=0,
\end{array}\right\}
$$

where $i$ may denote any term of the series $0,1,2,3, \ldots$

And thus, to take the first example which suggests itself, we see that there cannot subsist

$$
\left(\frac{1}{(a-b)^{2}}\right)\left(\mathrm{a} b^{\prime}\right)=\left(\frac{1}{a^{2}}\left\{1+\frac{2 b}{a}+\frac{3 b^{2}}{a^{2}}+. .\right\}\right)(a . . .)
$$

both when $b=a$, and when $b=b$. For the series which constitutes a factor of the equation of condition will be essentially divergent in one of these two cases : so that this equation will no longer be satisfied in virtue of the other factor $h\left(\begin{array}{l}h \\ 0\end{array}\right)$. 
degree may be reduced, as I showed some years ago in my Mathematical Researches*.

Long Stratton, Norfolk, May 1, 1845.

LXXXI. On the Distilled Waters of our Pharmacopoias. By Robert Warington, Esq.†

THE subject of flavoured waters, prepared by distillation or extemporaneously, through the medium of carbonate of magnesia, has for sonse time past engaged my attention; and as it is a question of considerable importance to the pharmaceutical chemist, I am induced to lay the detailed investigation before the Society.

The formulø given for the preparation of distilled waters in the London Pharmacopoia of 1836 is as follows:-To a specified quantity of material, whether it be essential oil, flowers, herbs, bark or berries, let 2 gallons of water and 7 oz. of proof spirit, having a specific gravity 0.920, be added, and submit the mixture to the process of distillation until one gallon shall have passed over. Presuming that the entire amount of spirit employed passes over in this operation, the resulting product will contain a quantity that will be equivalent to about $4 \frac{1}{2}$ ox. of rectified spirit of spec. grav. 0.838.

The Edinburgh form has rather less spirit to the proportion of materials, 2 gallons of water and 3 oz. of rectified spirit are so employed, and a gallon distilled.

The Dublin formula differs from the foregoing, in ordering half an ounce of rectified spirit to be added to each pound of the water after distillation, or in the ratio of $5 \mathrm{oz}$. to the gallon.

In the course of other investigations, some years since, $I$ founcl that when a very small quantity of alcohol was added to distilled water, and the mixture kept exposed to the air for a length of time, the containing vessel being carefully covered with paper to exclude the dust, it became gradually converted into acetic scid, and, by analogy, I was led to believe that the same effect would take place in these distilled waters; the following experiments were therefore put in operation to test the accuracy of these ideas.

In the distillation of essential oils, it is well known that the water which passes over is opalescent from its being highly charged with the oil, and that when thus impregnated, it will keep perfectly sound for a great length of time. Distilled waters were prepared from various of the essential oils without

- See Bir W. R. Hamilton's Inquiry on the subject in the Bixth Report of the British Association for the Advancement of Science.

† Communicated by the Chemical Society; having been read January 20,1845 . 